Published in International Journal of Rail Transportation (TJRT), accepted 16/12/16,

DOI: $10.1080 / 23248378.2016 .1274685$

The data from this paper is available at http://doi.org/10.5258/SOTON/404268

\title{
Anechoic wind tunnel tests on high-speed train bogie aerodynamic noise
}

\author{
Eduardo Latorre Iglesias, ${ }^{\mathrm{a}, *}$, David J. Thompson ${ }^{\mathrm{a}}$, Malcolm Smith ${ }^{\mathrm{a}}$, Toshiki \\ Kitagawa ${ }^{\mathrm{b}}$, Nobuhiro Yamazaki ${ }^{\mathrm{b}}$
}

${ }^{a}$ Institute of Sound and Vibration Research, University of Southampton, Southampton SO17 IBJ, UK; ${ }^{b}$ Railway Technical Research Institute, 2-8-38 Hikari-cho, Kokubunji-shi, Tokyo, Japan 185-8540.

*Corresponding author, tel: +34 655399 849, email: eduardolatorreiglesias@ gmail.com

Aerodynamic noise becomes a significant noise source at speeds normally reached by high-speed trains. The train bogies are identified as important sources of aerodynamic noise. Due to the difficulty to assess this noise source carrying out field tests, wind tunnel tests offer many advantages. Tests were performed in the large-scale low-noise anechoic wind tunnel at Maibara, Japan using a 1/7 scale train car and bogie model for a range of flow speeds between 50,76, 89 and $100 \mathrm{~m} / \mathrm{s}$. The dependence of the aerodynamic noise from the bogie region on different factors has been studied for different bogie configurations and inflow conditions representing different positions of the bogie along the train. The speed dependence and the noise directivity have also been assessed. The results show the particular importance of the components exposed to the free flow whereas those shielded within the bogie cavity are shown to radiate much less noise.

Keywords: aerodynamic noise; railway noise; high speed; wind tunnel, bogie. 


\section{Introduction}

Aerodynamic noise becomes a significant source of railway noise at speeds above $300 \mathrm{~km} / \mathrm{h}$ [1], which is now reached by many high-speed trains in commercial operation. The train bogie region is considered to be an important source of aerodynamic noise from high-speed trains, its significance being increased due to the fact that there are many bogies along the length of the train. Bogie aerodynamic noise is produced due to the complex turbulent flow recirculation around the bogie area [2]. A better understanding of the generation mechanism of bogie aerodynamic noise may allow to improve current prediction models and to develop noise control measures.

A common way to assess the different noise sources on a train is to use field tests. Microphone arrays, combined with signal processing techniques such as beamforming, are often used during these tests to localise and to quantify the contribution of different noise sources in the train. As an example, for a train speed of $350 \mathrm{~km} / \mathrm{h}$, Mellet et al. [3] found that the noise from the bogie region could be identified as broadband with a relevant contribution to the A-weighted level between $315 \mathrm{~Hz}$ and $2 \mathrm{kHz}$ and a speed exponent of 4.8. They found that the contribution of the bogies on the front power car is higher than that from the rear power car, showing the importance of the position of the bogie along the train in the aerodynamic noise generation. However, the separation of the rolling and aerodynamic noise from the bogie is not trivial and it is one of the main problems associated with such field tests. Mellet et al. [3] and Poisson et al. [4] proposed a method to discriminate aerodynamic and mechanical sources based on a study of their speed dependence. Nevertheless they pointed out the difficulty of the separation of these two sources. Fremion et al. [5] used Neise probes and microphones around the intercoach gap and bogie region and found that the aerodynamic noise produced in the train bogie region is generated by several uncorrelated 
acoustic sources rather than a global mechanism describing the radiation from the whole bogie.

Some other drawbacks are found when performing field tests that should be taken into account during the data analysis: the relative motion between the source and receiver produces a Doppler shift in the frequency of the received sound; the short duration of the signal that can be acquired and the unsteadiness of the noise sources also present difficulties. However, the main drawback of such field tests is the high cost associated with assessing new train prototypes or the effect of modifications in some components of the train.

An alternative to field tests is to perform noise tests in a wind tunnel. Closed-section wind tunnels are often used for aerodynamic measurements but high background noise and reflections from the walls make it difficult to perform noise tests. For this reason anechoic open-jet wind tunnels are preferred as the test section is surrounded by an anechoic chamber with sound absorptive walls, avoiding sound reflections, and attenuators are used on the inlet ducts to reduce the background noise. Additionally, most of the drawbacks associated with field tests are avoided. The use of scale models makes it more feasible to try different experimental set-ups and it is relatively easy to carry out parametric studies. However, some precautions are necessary when performing noise tests in an anechoic open-jet wind tunnel, which are explained in Section 2.

Some examples are found in the literature of anechoic open-jet wind tunnels used to assess the noise from different train components such as pantographs. However, studies of the aerodynamic noise from train bogies are rare. Lauterbach et al. [6] performed tests in an anechoic open-jet wind tunnel using a 1/25 scale Inter City Express 3 high-speed train focusing on the noise radiated by the pantograph and the bogie region. By performing the tests in a cryogenic wind tunnel, where the air density can be changed, an increase of the Reynolds numbers by a factor of 8 up to $3.7 \times 10^{6}$ could be reached. They found the noise 
from the bogie area to be dominated by cavity noise and to be significant for full-scale frequencies below $200 \mathrm{~Hz}$. Yamazaki et al. [7] performed wind tunnel tests on the noise radiated by a $1 / 7$ scale train bogie installed in the bogie cavity of a 1/7 scale high-speed train car model. They assessed the noise radiated by different configurations of a simplified bogie model. They also developed a method to assess the effect of the bogie position along the train taking into account the vertical flow speed profile at the inlet of the bogie cavity. This method was later extended taking into account the horizontal flow speed profiles at the inlet of the bogie cavity [8]. A more detailed review on modelling and experiments on aerodynamic noise from train bogies, pantographs and other sources is given by Thompson et al. [9].

A series of noise measurements are presented, carried out in the large-scale low-noise anechoic wind tunnel at Maibara, Japan using a 1/7 scale train car and bogie model. Information about the uniformity of the flow speed, flow turbulence intensity and background noise of this wind tunnel can be found in [10]. The effect of the incident boundary layer was not considered. However, as the bogie area is placed far from the train nose, its effect on the noise from the bogie area is not expected to be significant. The aim of this investigation is to study the aerodynamic noise radiated by the bogie region under different conditions. Variations are considered in the bogie configuration, inflow conditions and relative position of the bogie components in the cavity. The directivity of sound radiation is also determined. Results are presented for a range of flow speeds between 50 and $100 \mathrm{~m} / \mathrm{s}$. The results can be used for the calibration and validation of numerical and empirical prediction models.

Section 2 describes the experimental set-up used. Section 3 presents the various bogie cavity arrangements considered from which the background noise and noise from the bogie cavity can be assessed. Section 4 describes the various bogie configurations and includes the results obtained from the assessment of the bogie noise. Finally, Section 5 gives a simplistic 
method to assess the variation of the bogie aerodynamic noise due to changes in position of the bogie components with respect of the cavity.

\section{Experimental set-up}

\subsection{Train and wind tunnel arrangement}

A 1/7 scale train model, already used by RTRI for previous experiments [7, 8], was made available and was used for these experiments. It has the length of 1.5 vehicles of a standard high-speed train, it does not include an inter-coach gap and has only one bogie cavity. The position of the bogie cavity corresponds to the rear cavity of the leading vehicle. The train model is made of stiff wood and the exterior surfaces were painted and polished to obtain a smooth surface free of irregularities. Figure 1 shows a sketch of the train model and its dimensions and relative position with respect of the outlet nozzle of the wind tunnel.

A microphone array was located at a lateral distance of $3.5 \mathrm{~m}$ from the train centreline. The array is described in more detail in Section 2.3. Its centre was located $392 \mathrm{~mm}$ downstream with respect to the geometrical centre of the bogie model to account for flow convection effects. This distance was calculated for a flow speed of $320 \mathrm{~km} / \mathrm{h}(89 \mathrm{~m} / \mathrm{s})$ by using the method proposed by Amiet [11], in order to account for the effect of the shear layer on the propagation path of noise from the bogie area to the receiver. The convective amplification due to the relative motion between the air and the noise source was accounted for only in the directivity measurements (see Section 4.5), while the effect of the energy spread into adjacent frequency bands after passing through the turbulent shear layer [12-14] was not taken into account. 

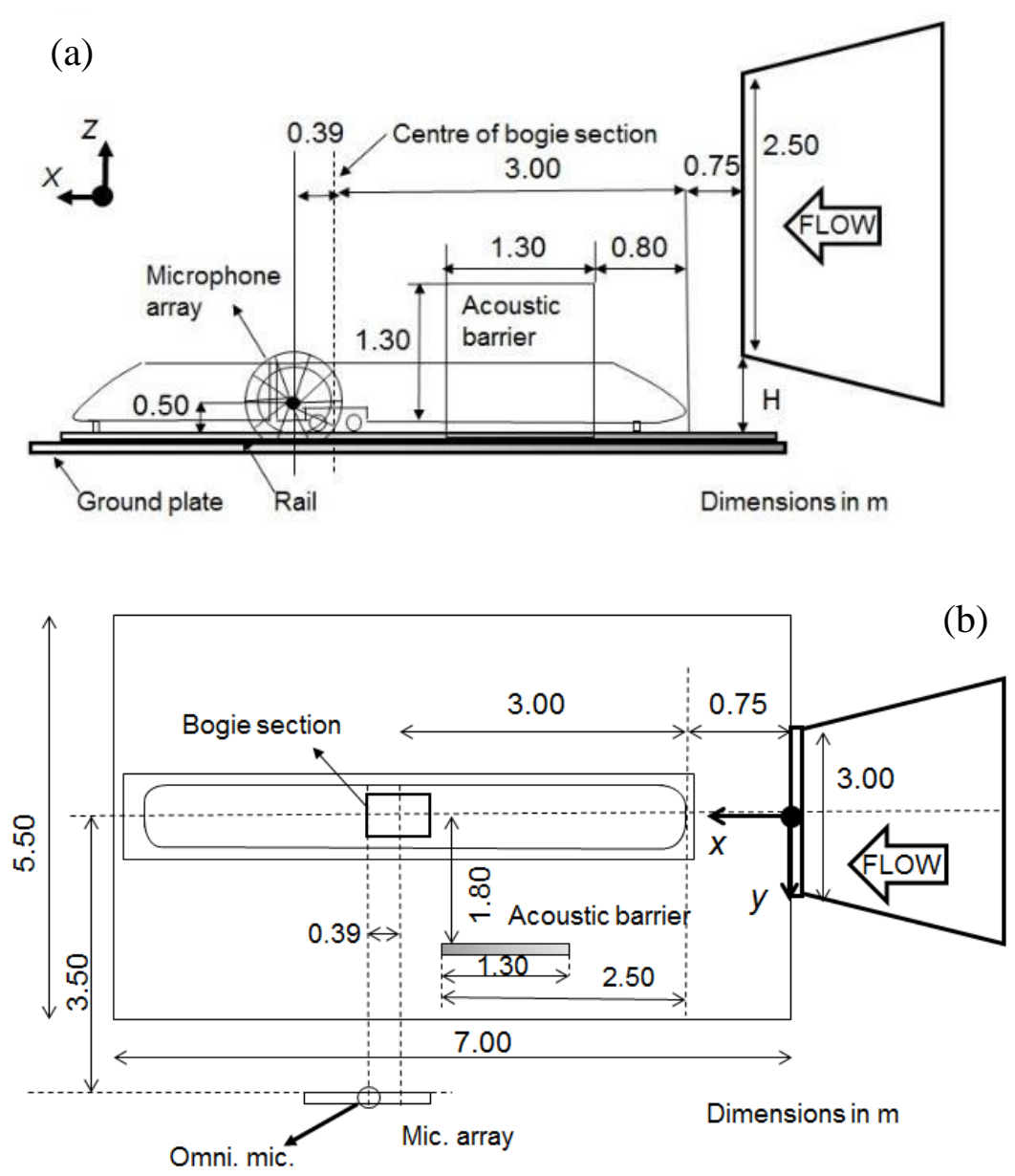

Figure 1. Experimental set-up. (a) Side view. (b) Plan view (not to scale).

Figure 2 shows a general view of the train model in the wind tunnel and of the experimental set-up. The $1 / 7$ scale train car body was attached above a reflective ground. The train model was mounted on simplified rails with a rectangular cross-section of size 28.5 by $19 \mathrm{~mm}$ fixed on the ground plane. The vertical position of the ground plane relative to the wind tunnel inlet could be adjusted. The wind tunnel open section is located inside an anechoic chamber of dimensions $20 \mathrm{~m}$ x $22 \mathrm{~m}$ x $13 \mathrm{~m}$.

The microphone array was used in order to increase the signal-to-noise ratio, defined as the ratio between the noise radiated by the bogie and by the other noise sources present in the experiments (e.g. train nose, wind tunnel self-noise...). In addition, a simple noise barrier was installed between the microphone array and the nose of the train to minimize the 
influence of noise sources located in that area. An omnidirectional microphone was placed in the geometrical centre of the microphone array and was used as a reference.

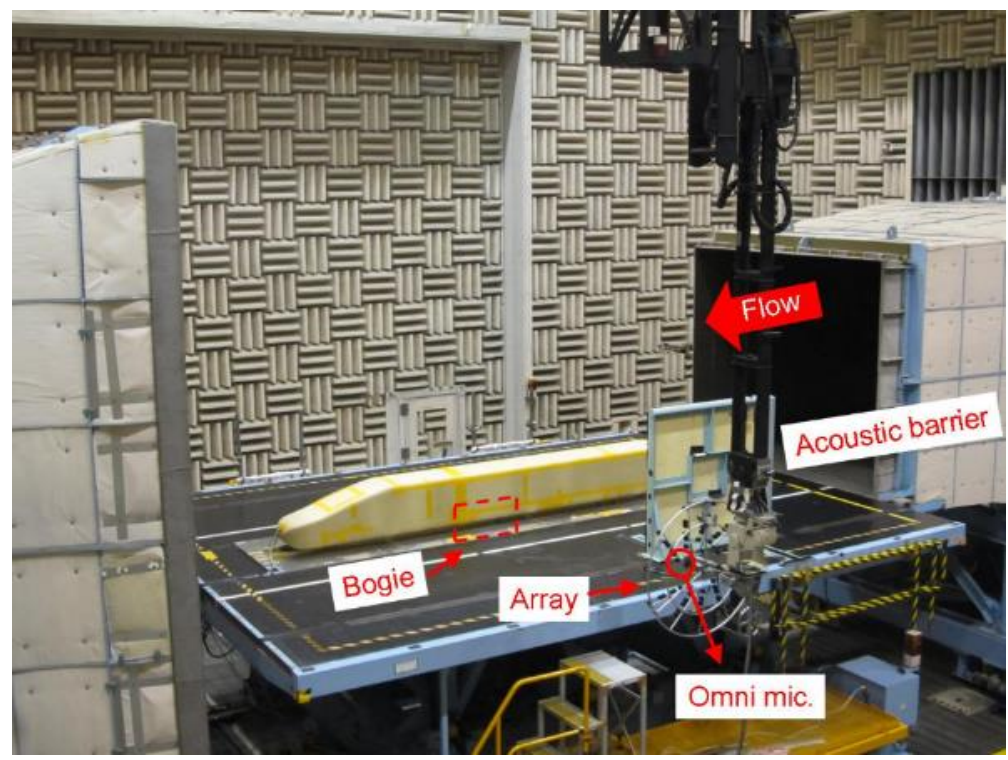

Figure 2. General view of the experimental set-up for the microphone array configuration.

\subsection{Scale models and frequencies}

Some consideration has to be given to the use of a scale model mock-up. Firstly, different Reynolds numbers can be expected compared with the real case, which can lead to different flow behaviours around the model (the Reynolds number is defined as $R e=U D / v$, where $U$ is the flow velocity, $D$ is a characteristic length and $v$ is the kinematic viscosity). In this case the flow speeds used, between 50 and $100 \mathrm{~m} / \mathrm{s}$, are in the range of train speeds normally reach by high-speed trains but the size is seven times smaller.

Another factor to be considered when scale models are used is the change in frequency. The Strouhal number $S t=f_{0} D / U$, where $f_{0}$ is the vortex shedding frequency, can normally be considered to be independent of the scale factor. If the size of the mock-up is smaller than the real component then the vortex shedding peak will be displaced to higher frequencies. Therefore, if scale models are used for noise tests the frequency should be converted to full scale by dividing the measured frequencies by the scaling factor. In the present case, the frequency range has been converted to full scale by dividing the narrow 
band frequencies by a factor of 7 . The SPL is presented in $1 / 3$ octave frequency bands between 125 and $3150 \mathrm{~Hz}$ full-scale $(875 \mathrm{~Hz}$ and $22 \mathrm{kHz}$ at model scale). For full-scale frequencies below $125 \mathrm{~Hz}$ the wind tunnel background noise was too high.

Finally, the difference in the size of the mock-up and the real component will also generate differences in the amplitude of the aerodynamic noise generated, because this is proportional to the surface area of the body placed in the flow [15]. However, in the present results the amplitude of the measured noise was not scaled to allow for this.

\subsection{Microphone array}

For most of the bogie configurations used during the tests the background noise caused by the wind tunnel and the other aerodynamic noise from the train model caused the signal-to-noise ratio measured by a single omnidirectional microphone to be poor especially under the middle car condition. The background noise masked the noise radiated by the bogie in most cases except those with the side frame extended (see Section 4.4) [16]. For this reason the noise spectra were estimated from the data measured by the microphone array using beamforming techniques.

The microphone array used during the tests consists of 66 microphones in a spiral arrangement (see Figure 2). The height of the centre of the array with respect to the reflecting ground plane where the train was installed was $0.5 \mathrm{~m}$. The outer diameter $\mathrm{D}_{\text {array }}$ was $1 \mathrm{~m}$ and the minimum distance between microphones $\mathrm{d}$ was around $3 \mathrm{~cm}$. The minimum frequency for which two separated monopole sources can be separated is given approximately by $\mathrm{f}_{\min }=$ $\mathrm{c}_{0} / \mathrm{D}_{\text {array }}$, where $\mathrm{c}_{0}$ is the sound speed, and the maximum frequency without incurring spatial aliasing is given by $f_{\max }=c_{0} / 2 d[17]$, in this case this gives $f_{\min }=340 \mathrm{~Hz}$ and $f_{\max }=5600 \mathrm{~Hz}$ as measured for the $1 / 7$ scale bogie model or $f_{\min }=49 \mathrm{~Hz}$ and $\mathrm{f}_{\max }=800 \mathrm{~Hz}$ if frequency is converted to full-scale. In the present experiments a full-scale frequency range from $125 \mathrm{~Hz}$ 
to $3150 \mathrm{~Hz}$ is covered so the upper limit is exceeded. However, the spiral arrangement of the microphones in the array minimises the impact of spatial aliasing on the results up to frequencies above this upper frequency limit [17]. A camera was installed in the centre of the microphone array in order to capture images to be used with noise maps for source localization.

For the calculation of the noise spectrum from the microphone array output, the method proposed by Yamazaki et al. is used [8]. The two-dimensional distribution of the noise in the bogie region was obtained from the microphone array data by applying a standard delay-and-sum beamforming algorithm. A grid of $20 \times 20$ values of sound pressure level (SPL) covering an area around the bogie region (with spatial resolution of $0.1 \mathrm{~m}$ in both horizontal and vertical directions) was calculated for each frequency band. The source position was localized by finding the cell of the grid containing the maximum SPL. Then, the values of the squared pressure were summed over an area of $7 \times 7$ cells around the cell with the maximum value. This area was estimated to cover all the significant values. This process was repeated for each frequency band. A frequency dependent correction factor was then applied to the data to account for the effects of array directivity. This factor was obtained by measuring the noise spectrum using an omnidirectional microphone located at the centre of the array as well as the corresponding array output for a reference noise source that provides a high enough signal-to-noise ratio under the bogie condition without side covers. This reference case consisted of the motor bogie with the lateral frame extended by $100 \mathrm{~mm}$ (see Section 4.4).

In all cases the location of the dominant noise source was found to be independent of the frequency band of analysis, showing the broadband characteristic of the noise spectrum radiated by the bogie components [16]. The noise maps in each case are shown only for the $1 / 3$ octave frequency band of $1 \mathrm{kHz}$ as this provides a good compromise between spatial 
resolution and signal-to-noise ratio. Moreover, the analysis of the noise maps for different flow speeds did not show any significant differences [16]. For this reason, the noise maps are presented only for the flow speed of $89 \mathrm{~m} / \mathrm{s}$.

\subsection{Inflow conditions}

The inflow conditions have a significant effect on the radiated noise. In this case the train bogie is installed in the bogie cavity of a train car body in order to approximate the inflow conditions of the real case. Another factor to be considered is that in the real case the train is moving and the ground is stationary. This relative movement can be simulated in wind tunnel tests using a moving belt under the train but this has not been used here as it would generate additional background noise. Flow speed measurements carried out by Iwasaki et al. [18] during a field test campaign were used to account for the absence of moving ground in the bogie inflow conditions. They measured the mean flow speed in the sleeper direction at different positions at the bogie cavity inlet of the rear bogie of the first car (leading) and the rear bogie of the fifth car (middle) of a real train on a slab track [18]. A rake of Pitot tubes was installed on the ground at fixed positions. They found that the flow speed measured for the leading car was significantly higher than that measured for the $4^{\text {th }}, 5^{\text {th }}$ and $6^{\text {th }}$ cars, these being similar to each other.

Following the procedure used by Yamazaki et al. [8], the set-up used in the wind tunnel tests was modified to obtain flow speed profiles at the inlet of the bogie cavity as similar as possible to those obtained by Iwasaki et al. during their field tests. Two different set-up configurations were used for the 'leading' and 'middle' car flow considerations (more details are given in [8]):

- Leading car: the rails were broadened locally by including two additional pieces upstream of the bogie cavity. 
- Middle car: the rails were kept without modifications but the ground plate was lowered by $175 \mathrm{~mm}$ relative to the inlet nozzle.

The flow speed profiles at the bogie cavity inlet were measured using a rake of Pitot tubes, as shown in Figure 3. The effect of the Pitot tube supports on the flow measurements was not accounted for. This arrangement allows the local mean flow speed to be measured at different distances from the train floor (heights below the floor between 1 and $53.5 \mathrm{~mm}$ in steps of $7.5 \mathrm{~mm}$ ) and at different distances from the centre of the bogie cavity (from 0 to 244 $\mathrm{mm}$ in several steps of typically $56 \mathrm{~mm}$ ). The position of each of the measurement points is indicated in Figure 4; the measurement plane was located upstream of the bogie cavity inlet. As flow symmetry was assumed, the speed profiles were only measured at one side of the centreline of the train model.

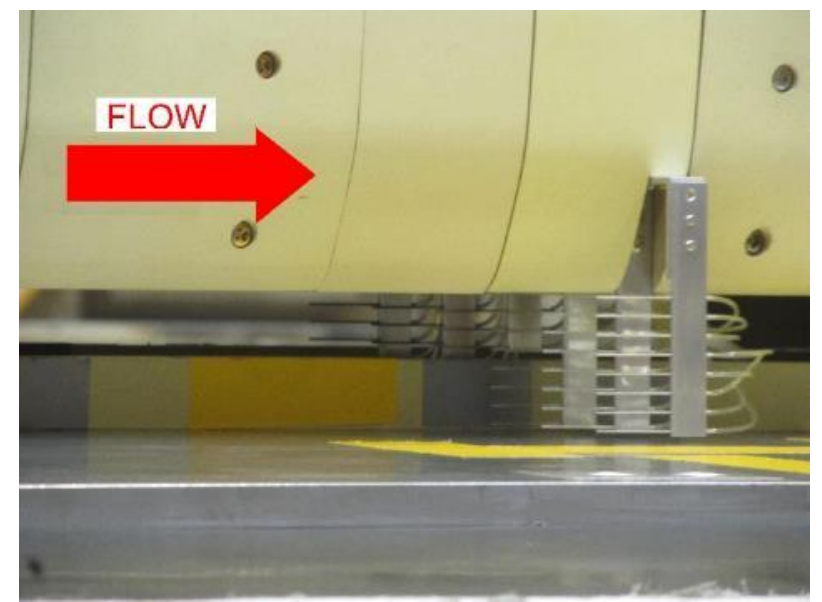

Figure 3. Rake of Pitot tubes used to measure the flow speed profiles at the bogie cavity inlet.

Figure 5 shows the flow profiles along the transverse direction -just above the rail height- obtained in the present wind tunnel tests using a 1/7 scale model for a flow speed of $89 \mathrm{~m} / \mathrm{s}$ and those obtained by Iwasaki et al. during field tests with a full-size train [18]. The flow speed profiles are presented in terms of the normalised speed, defined as $U_{i} / U_{\infty}$ is where $U_{i}$ is the flow speed measured at each of the measurement points and $U_{\infty}$ is the mainstream 
flow speed. The position of the measurement points is normalised by the track gauge so the rail centreline is at 0.5 . Good agreement is found with the field measurements.

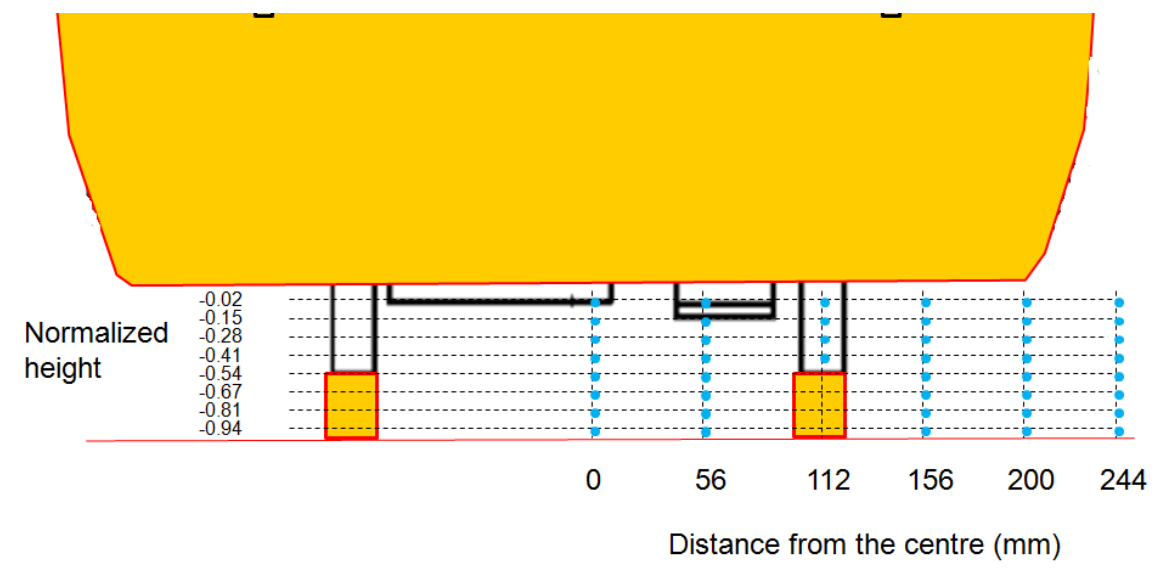

Figure 4. Sketch showing the positions of the bogie cavity inlet where the mean flow speed was measured.

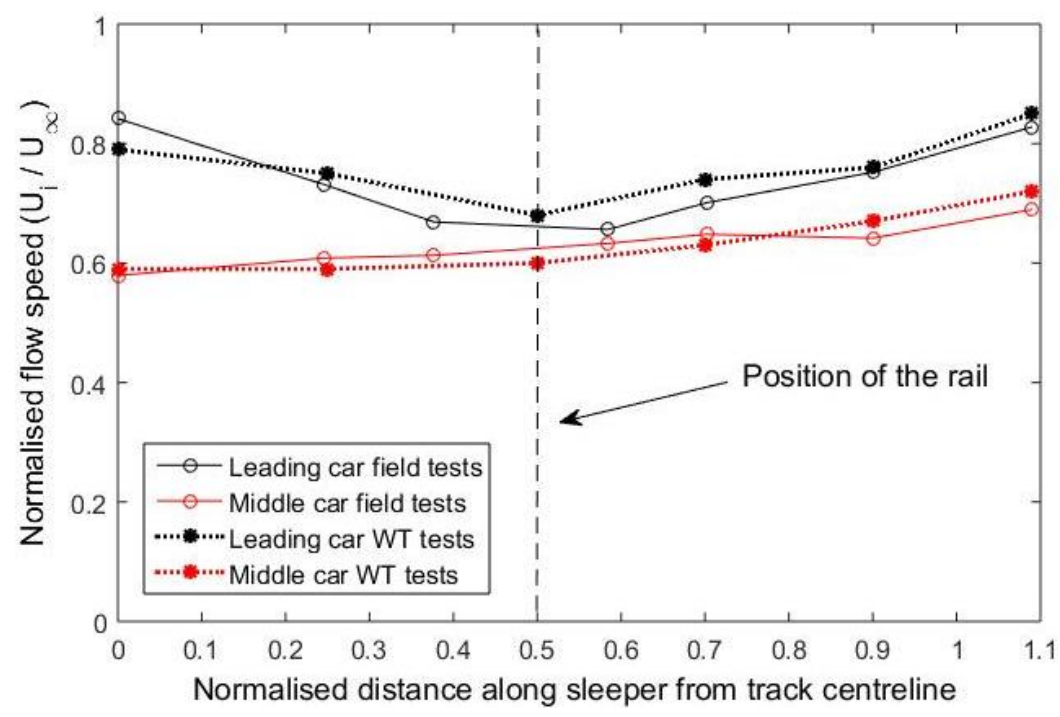

Figure 5 Transversal normalised flow speed measured at positions just above the rail. Comparison between the present wind tunnel tests and field test made by Iwasaki et al. [18]. The distance is normalised by the track gauge so the position of the rail centreline corresponds to 0.5 .

\section{Background noise and noise from the bogie cavity}

The present study is focused on the noise produced by the bogie. In order to understand the contribution of the different bogie components and the bogie cavity itself various configurations are studied. Figure 6 shows the different bogie cavity configurations used 
during the experiments. Figure 6(a) shows the original design of the bogie cavity with sharp edges. In Figure 6(b) a modified version of the bogie cavity is shown in which the downstream edge was rounded. In Figure 6(c) the bogie cavity has been closed using side skirts and an extension of the train floor, while in Figure 6(d) the bogie is installed in the bogie cavity together with the side skirts. Just the side frames mounted in the bogie cavity are shown in Figure 6(e) and the complete bogie model installed in the cavity is shown in Figure 6(f).

The configurations shown in Figure $6(\mathrm{~b}, \mathrm{c})$ can be considered as different estimates of the background noise. In the configuration BG shown in Figure 6(c), the bogie cavity was covered by side skirts and the train floor was extended under the cavity. This therefore excludes the noise generated by any sources in the bogie cavity region and gives a measure of the background noise from the wind tunnel and other parts of the train body. The configuration $\mathrm{C}$ shown in Figure 6(b) consists of the rounded bogie cavity with no bogie. It therefore includes the noise radiated by the bogie cavity when this is empty and it can be taken as the effective background noise, i.e. the noise due to all sources except the bogie, as the main interest of this investigation is to assess the noise produced by the bogie itself.

As shown in Figure 6(a), the original cavity of the 1/7 scale high-speed train car model had sharp upstream and downstream edges. In previous experimental studies [7], carried out in the same wind tunnel using a similar set-up, it was found that the noise produced by the interaction between the downstream cavity edge and the incident turbulent flow produced from the upstream cavity edge had a significant contribution to the overall noise radiated by the bogie area [7]. The downstream edge was therefore rounded (Figure 6(b)) in an attempt to decrease the interaction noise and increase the signal-to-noise ratio. 


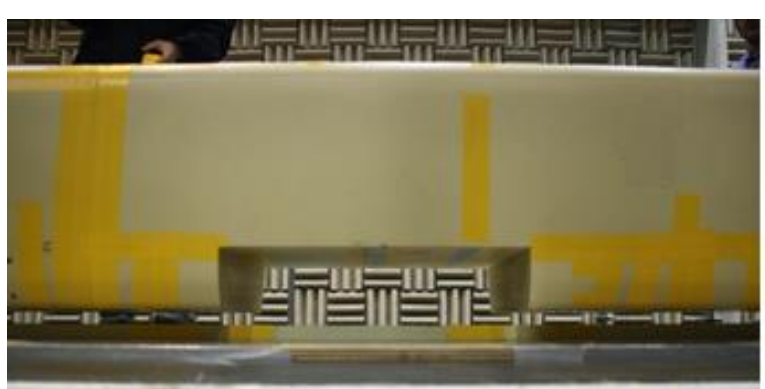

(a)
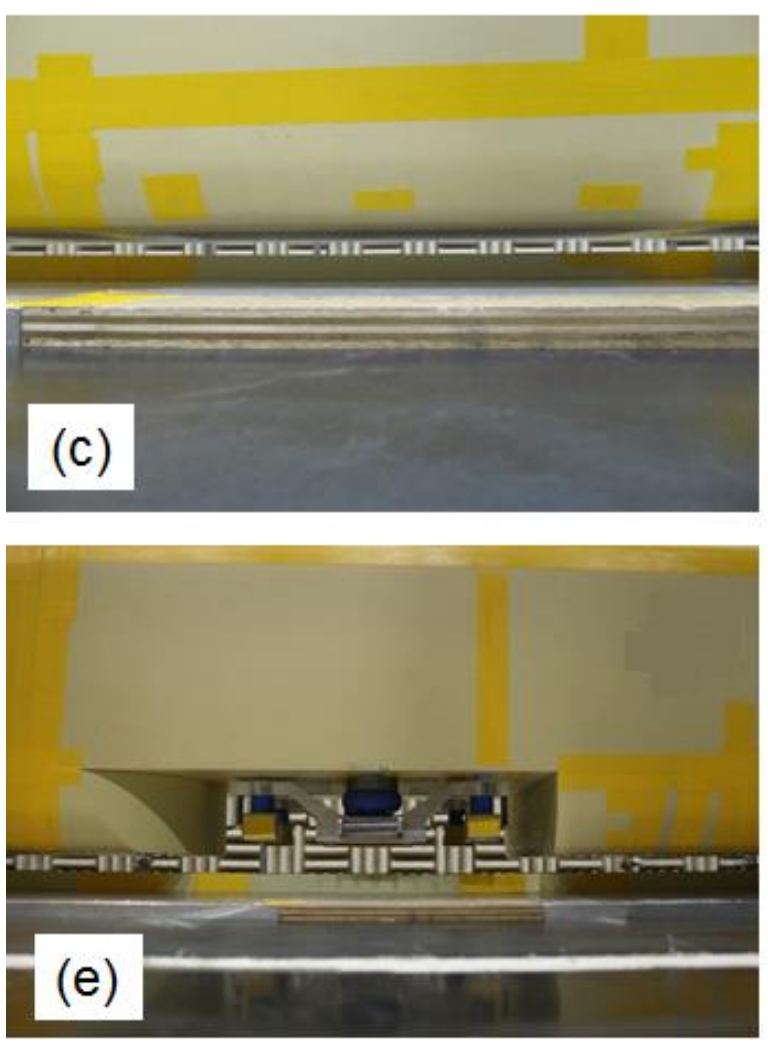
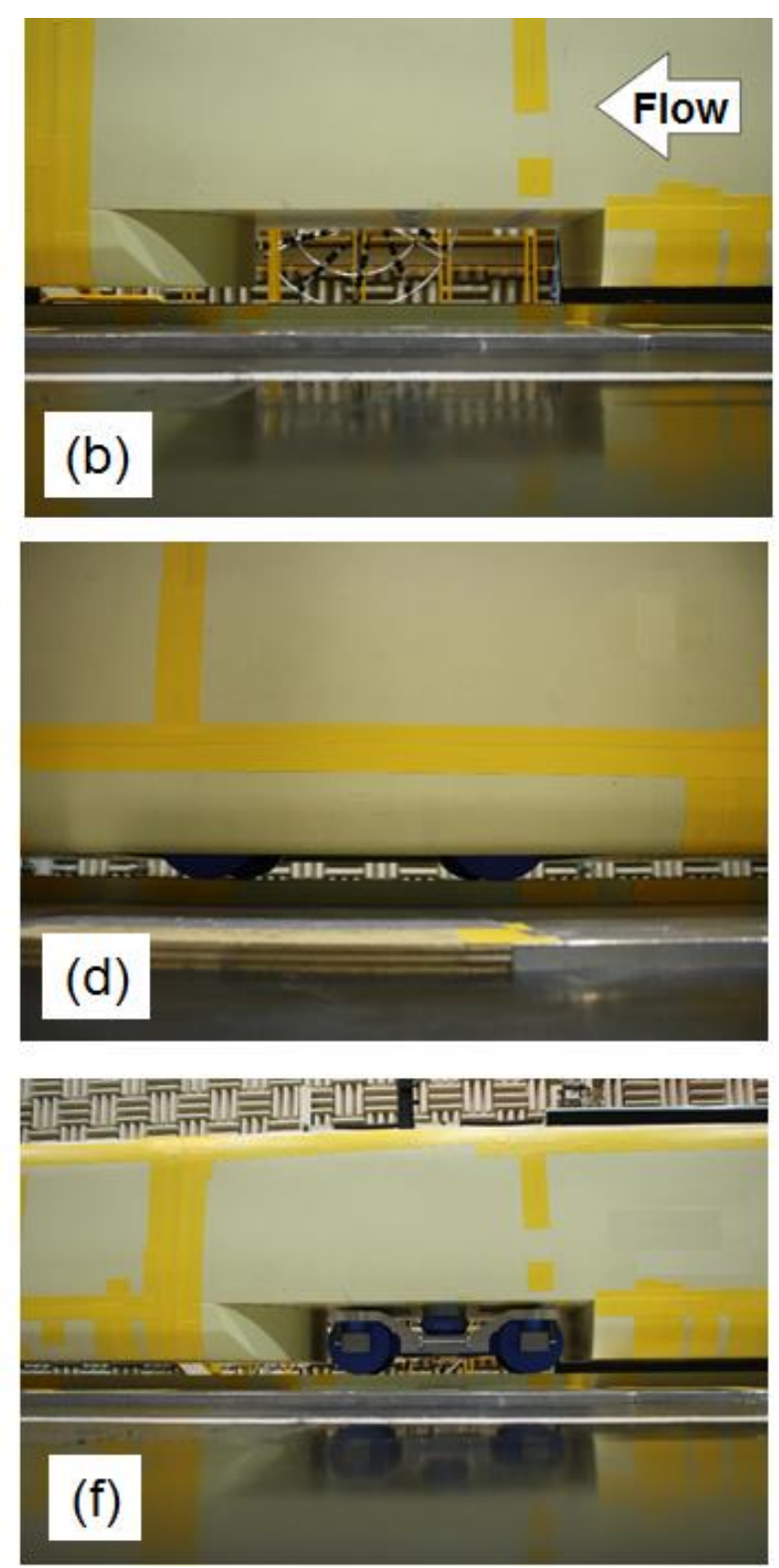

Figure 6. Views of the different bogie configurations used during the tests. (a) Cavity with sharp edges (C+sharp). (b) Cavity with rounded edges (C). (c) Empty cavity with side skirts and closed floor (BG). (d) Motor bogie and side skirts (MB+skirt). (e) Side frames (FR). (f) Complete motor (MB) or trailer bogie (TB).

Figure 7 shows various results for the leading car flow configuration and a speed of $89 \mathrm{~m} / \mathrm{s}$. Figure 7(a) shows that the noise from the cavity with sharp edges is significantly higher than that from the rounded version of it and is actually similar to the noise produced when the bogie is included in the cavity with rounded edges, shown in Figure 7(b). By rounding the downstream edge of the cavity the overall noise was reduced by $3.7 \mathrm{~dB}$, with 
reductions up to $7 \mathrm{~dB}$ in some of the frequency bands. The background noise generated by the configuration with the cavity filled in $(\mathrm{BG})$ is more than $5 \mathrm{~dB}$ lower than that measured for the configuration $\mathrm{C}$ for much of the frequency range. The noise decreases significantly when the noise cavity is covered (flat floor configuration in Figure 6(c)).

Figure 7(b) shows the noise spectra measured for the configurations with the empty rounded cavity (C) and the case with a typical bogie present (MB), again for the leading car flow configuration. The bogie configurations are described in more detail in Section 4. Despite the rounding of the cavity edges, the noise from the configuration $\mathrm{C}$ cannot be neglected. The background noise increases at high frequencies, reducing the signal-to-noise ratio. However, no correction for this was applied, despite the low signal-to-noise ratio at high frequencies.
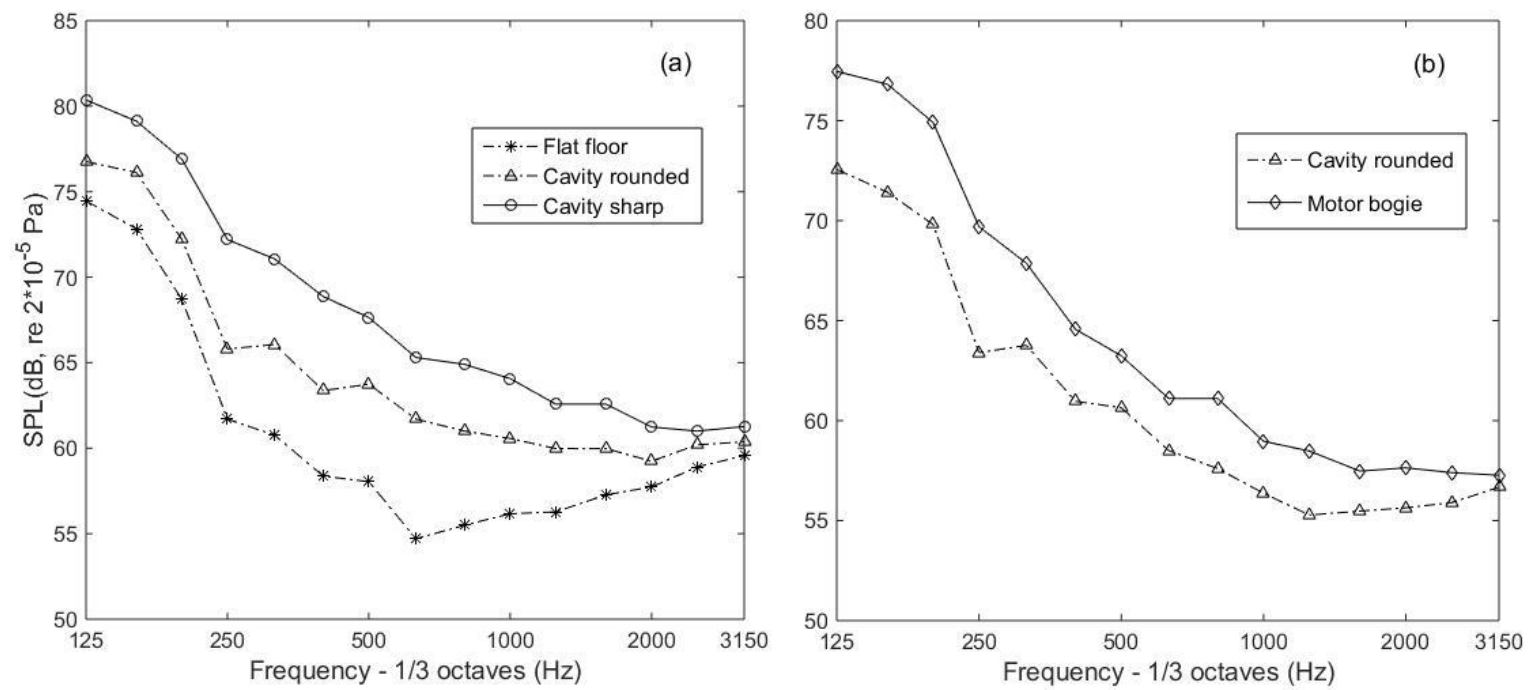

Figure 7. Comparison between the noise radiated by different configurations under leading flow conditions for a flow speed of $89 \mathrm{~m} / \mathrm{s}$. (a) Configurations BG, C and MB. (b) Configurations BG, C and C+sharp.

\section{Noise from the bogie}

\subsection{Bogie mock-ups}

A 1/7 scale mock-up of a simplified bogie was specifically designed for the current wind 
tunnel tests. Two different configurations of the bogie model were built to depict a simplified trailer and motor bogie, as shown in Figure 8. The trailer bogie included the front and rear brake systems, each with three brake discs made of stiff plastic, while the motor bogie had a front and rear motor and gear box made of wood.

The side frames, axles and shafts were made of aluminium. The design of the bogie mock-up allowed the side frames to be removed and the components attached to the axles to be exchanged. Therefore, the side frames could be used separately or combined with the bogie components to form the trailer and the motor bogie configurations.
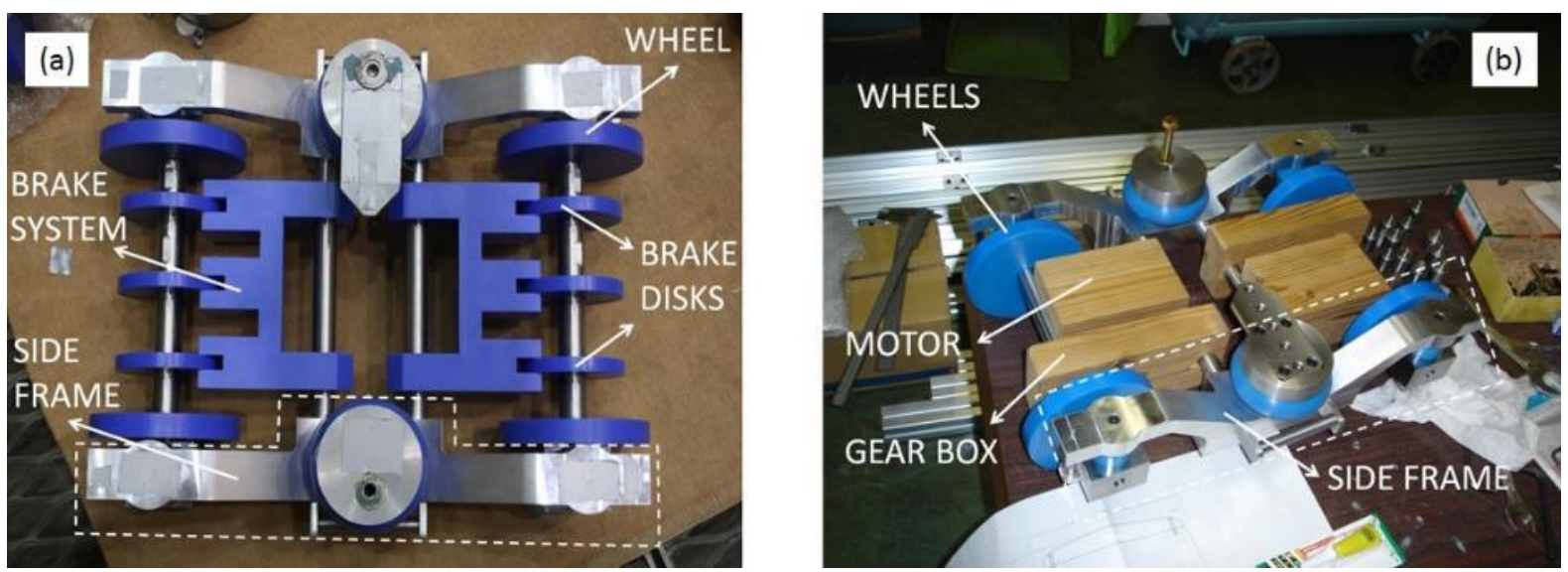

Figure 8. 1/7 scale simplified bogie mock-up. (a) Trailer bogie. (b) Motor bogie.

The bogie was attached in the bogie cavity beneath the train car body, see also Figure 6(f). Figure 9 shows a sketch of the bogie installed in the cavity where the main dimensions are given.

\subsection{Noise from different bogie configurations}

The noise spectra from the motor and trailer bogie configurations are compared in Figure 10. In addition, the result is shown for the side frames with no internal components, as shown in Figure 6(e). The noise spectra from the trailer and motor bogies are quite similar, with differences less than $2 \mathrm{~dB}$ in all the frequency bands. When the internal components (including wheels) are removed, however, the noise decreases significantly and the amplitude 
of the noise spectrum is very close to that radiated by the empty bogie cavity.

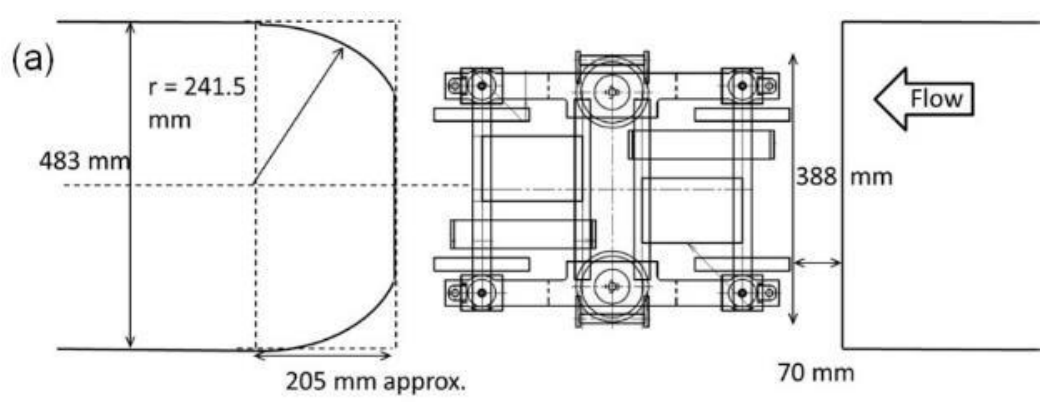

(b)

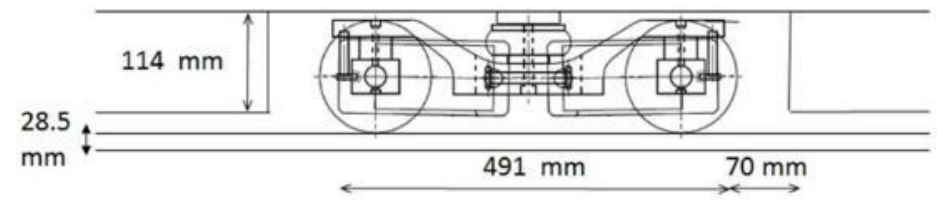

Figure 9. Sketch of the bogie mock-up installed in the cavity. Main dimensions are given. (a) Plan view. (b) Side view.

Figure 11(a) shows noise map for the motor bogie. This indicates that the noise, for $1 / 3$ octave frequency band of $1 \mathrm{kHz}$, is localized towards the bottom of the bogie region for both cases but it is not possible to identify a specific noise source. When only the side frames are present, as shown in Figure 11(b), the main source of noise is the cavity downstream edge, showing that no significant contribution is coming from the side frames (which agrees with Figure 10).

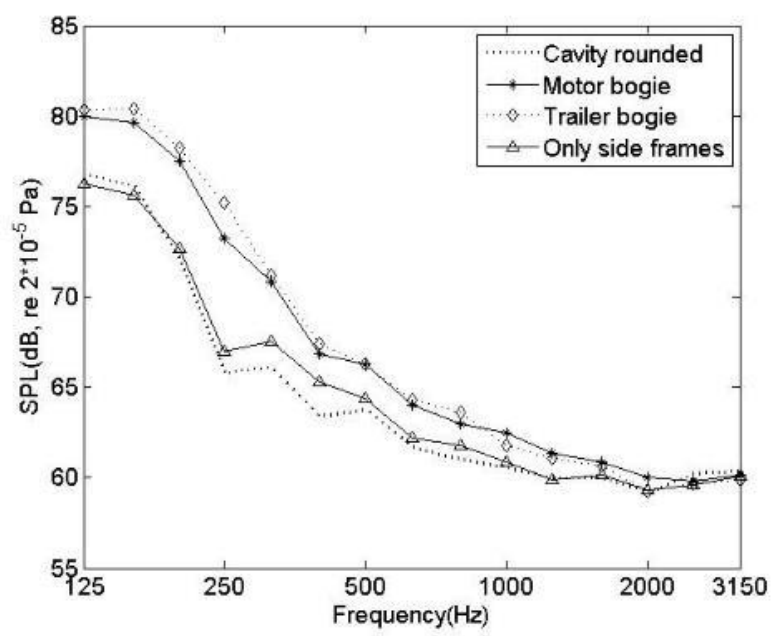

Figure 10. Comparison between the noise radiated by the motor bogie, the trailer bogie and just the side frames. Leading car flow configuration and flow speed of $89 \mathrm{~m} / \mathrm{s}$. 

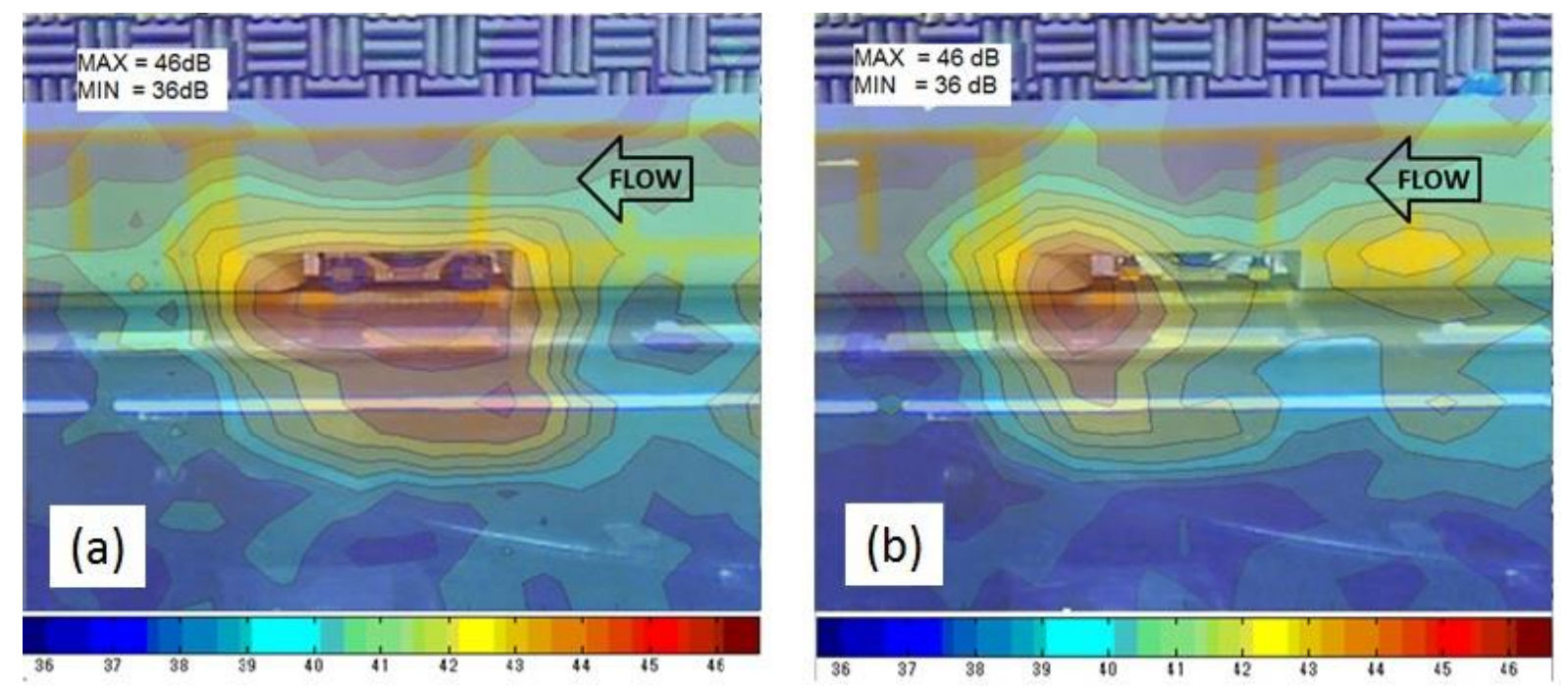

Figure 11. Noise maps for the full scale $1 / 3$ octave frequency band of $1 \mathrm{kHz}$ for the motor and trailer bogie configurations and for just the side frames. Flow speed of $89 \mathrm{~m} / \mathrm{s}$. (a) Motor bogie (MB). (b) Only frames (FR).

Figure 12 shows a sketch of the front view of the bogie cavity area from which the bogie components, or parts of them, which are fully exposed to the incoming flow can be identified. The wheels are the components with the largest surface area exposed to the incident flow.

The side skirts configuration, shown in Figure 6(d), leads to a noise reduction of about 2 to $3 \mathrm{~dB}$ that is nearly independent of frequency $[8,18]$. The skirts act as a barrier for the sound generated by the bogie and also shield the bogie components of the incoming side flow.

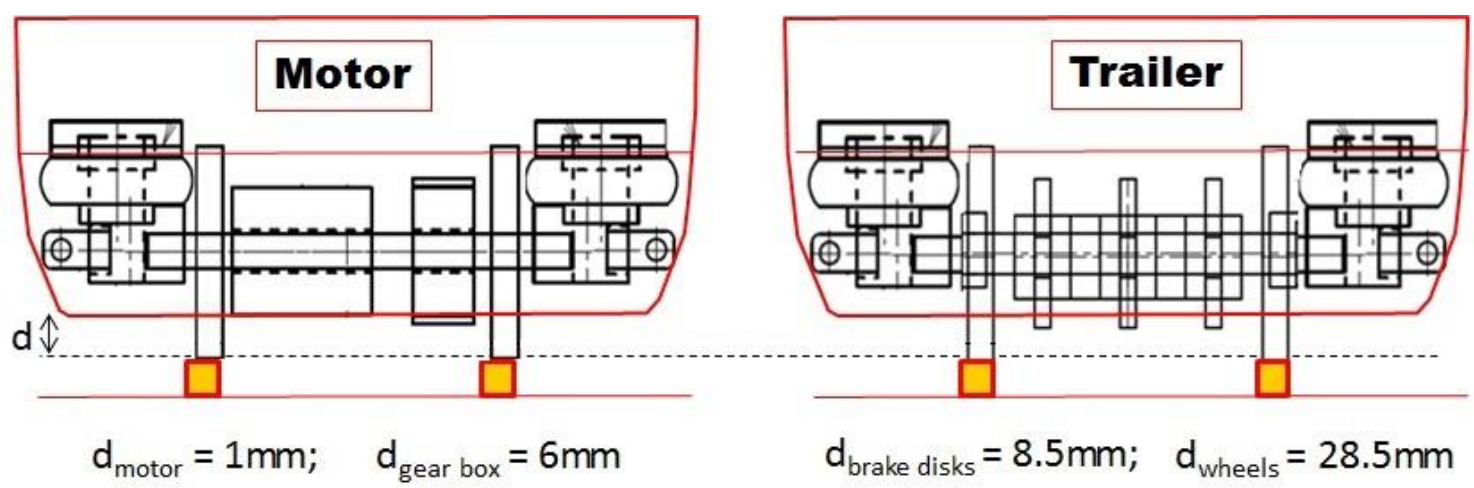

Figure 12. Sketch showing the frontal view of the motor and trailer bogie configurations. The height of the part of the components located outside the bogie cavity is given (reduced scale). 


\subsection{Dependence on the inflow conditions}

The position of the bogie along the train will modify the inflow conditions and as a consequence the noise radiated by the bogie. In this case the measured noise obtained for the inflow conditions approximating the rear bogie cavity of the leading and fifth ('middle') train cars are shown.

Figure 13(a) shows the noise spectra radiated by the motor bogie and the trailer bogie for these two flow configurations. Figure 13(b) shows the difference between the SPL radiated by each of the bogie configurations if the leading car and the middle car flow conditions are compared. The corresponding result for the empty cavity is also shown.
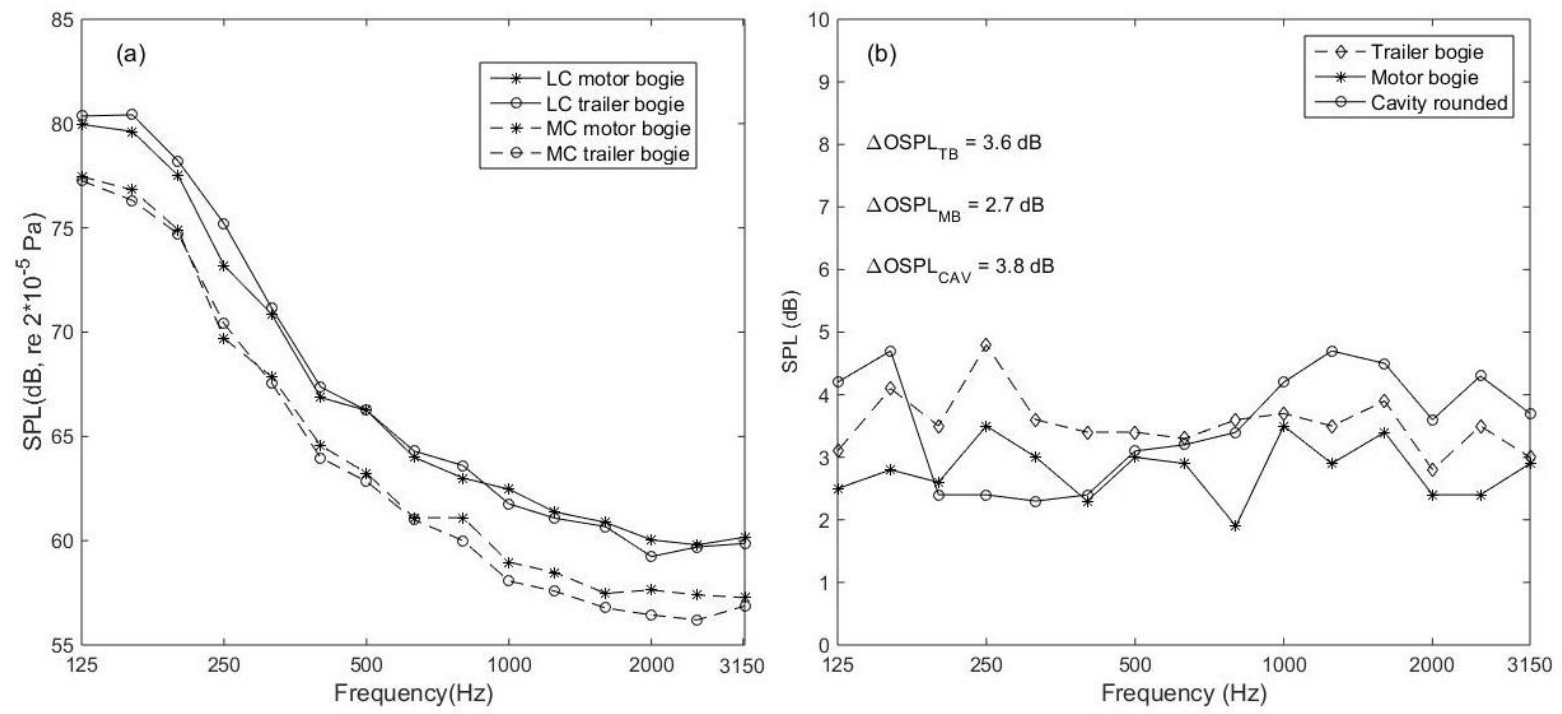

Figure 13. Motor bogie (MB), trailer bogie (TB) and cavity noise (C) for the leading (LC) and middle car (MC) flow configurations. Flow speed of 89 m/s. (a) Noise spectra. (b) Difference SPL (LC - MC).

The noise spectra are higher for the leading car flow configuration in all the cases tested, as expected due to the higher incident flow speed. The SPL difference obtained in the present experiments is relatively independent of frequency in each case, with a variability less than $+/-1.5 \mathrm{~dB}$. In terms of the overall SPL over the frequency range 125 to $3150 \mathrm{~Hz}$, the difference is very similar for the trailer bogie and cavity-only configurations ( 3.6 and $3.8 \mathrm{~dB}$ ) 
and is around $1 \mathrm{~dB}$ lower for the motor bogie configuration. These results are in good agreement with those obtained by Yamazaki et al. [8].

To help interpret these results, Figure 14 shows contour maps representing the normalised flow speed measured at different positions of the bogie cavity inlet for the leading and middle car flow configurations. From these results it can be inferred that the normalised flow speed is lower for the middle car than for the leading car for all the measurement points. The average reduction in speed is a factor of 0.83 . The lowest flow speeds are at the points close to the surfaces (ground and car floor). Moreover, the flow speed is reduced above the rail which corresponds to the position of the wheels. Outside the bogie cavity, at positions greater than $200 \mathrm{~mm}$ from the track centreline, the flow speed is higher than beneath the train.
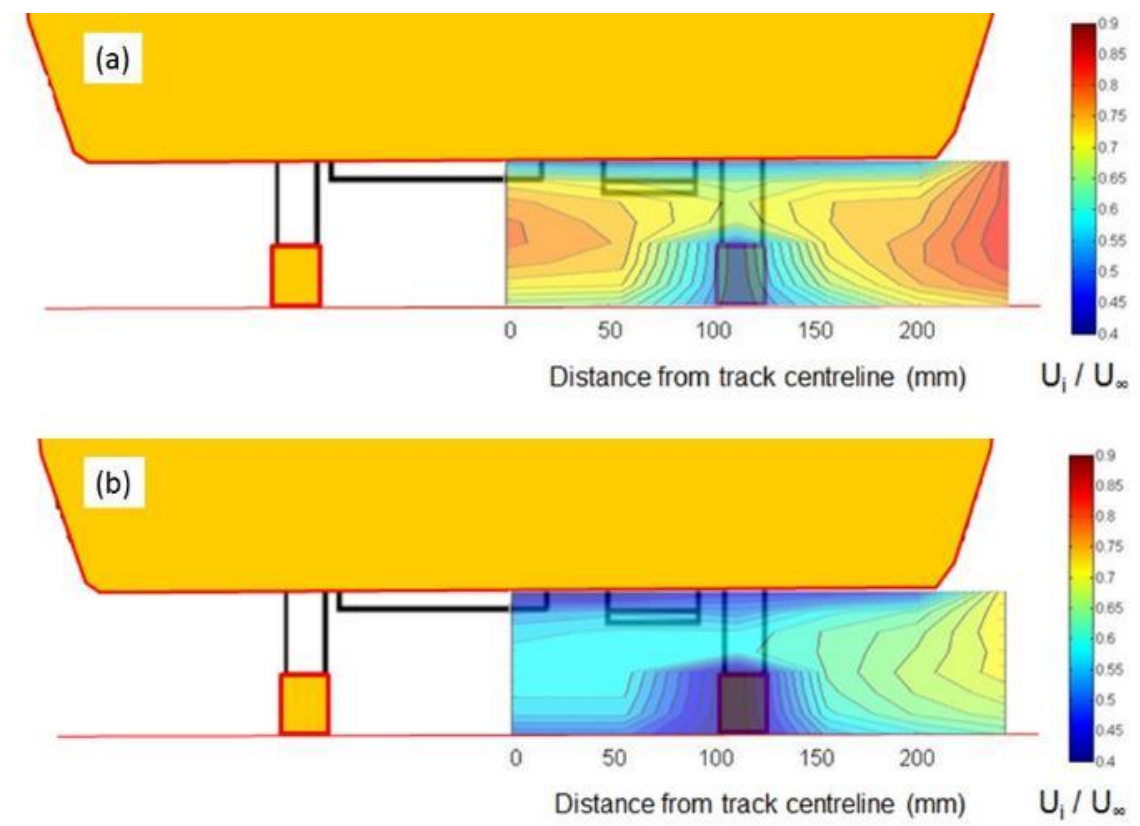

Figure 14. Contour map showing the normalized flow speed at the bogie cavity inlet. (a) Leading car flow configuration. (b) Middle car flow configuration.

\subsection{Noise from the bogie sides}

As seen in Figure 12, the bogie frame is almost entirely shielded within the cavity. However, the Japanese car body used for the scale model is wider than most trains used in Europe and 
particularly in the UK. Therefore a series of experiments have been carried out to vary the lateral position of the bogie side frame relative to the train body.

To achieve this the wheel shafts and transverse bogie shafts were made extendable allowing one of the side frames to be moved laterally. Three different shaft extensions were used: 50, 75 and $100 \mathrm{~mm}$. Figure 15(a, b) shows two different views of the motor bogie when the axles were extended by $100 \mathrm{~mm}$. Figure $15(\mathrm{c}, \mathrm{d})$ shows frontal and upper views of the bogie side when installed in the bogie cavity after extending the axles by $100 \mathrm{~mm}$. These configurations, which are based on the motor bogie $(\mathrm{MB})$ are denoted $\mathrm{MB}+\mathrm{S} 50, \mathrm{MB}+\mathrm{S} 75$ and $\mathrm{MB}+\mathrm{S} 100$.
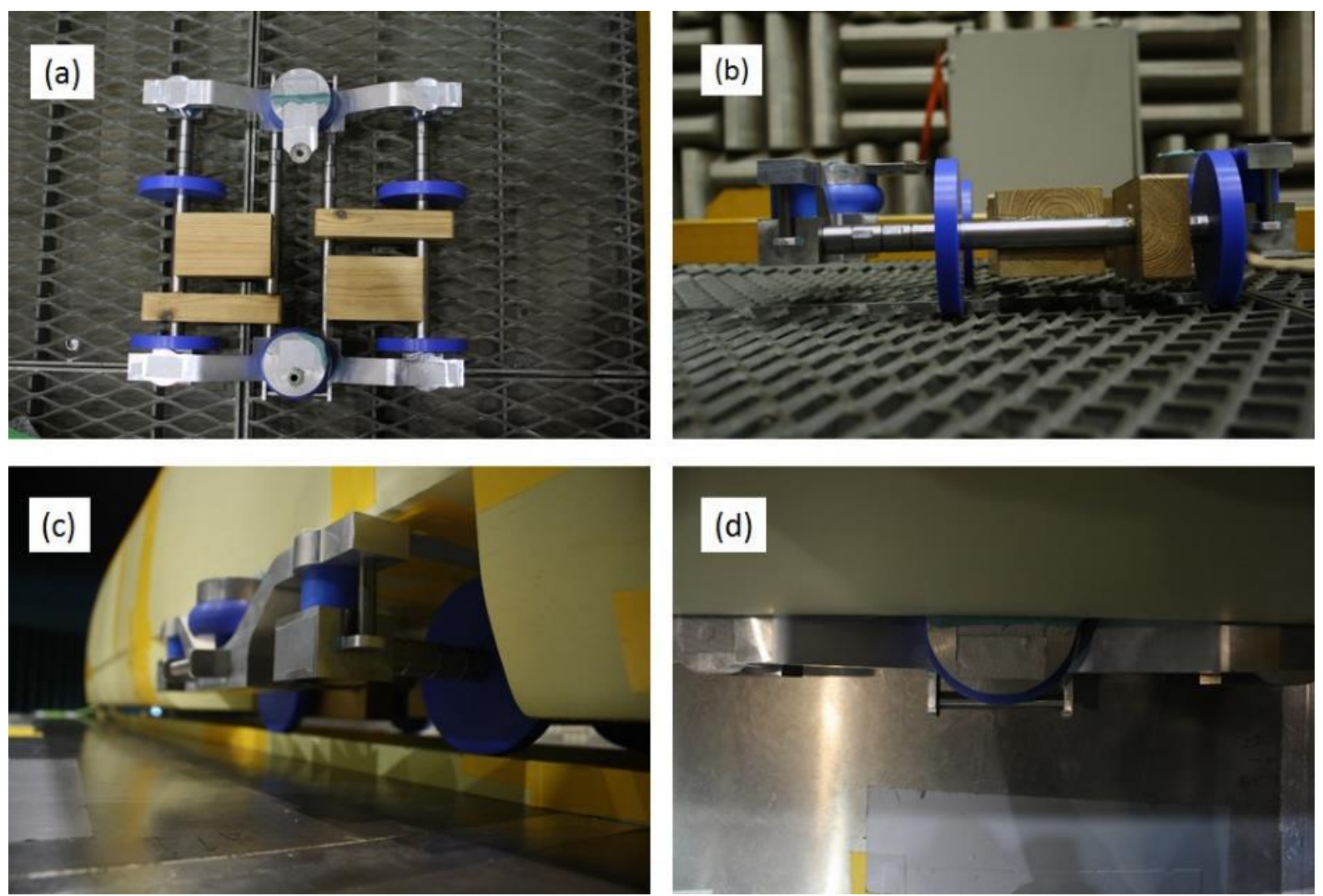

Figure 15. Motor bogie mock-up with the wheel shafts extended by $100 \mathrm{~mm}$. Bogie alone: (a) top view; (b) front view. Components of the bogie outside the bogie cavity: (c) front view; (d) top view.

When the axles were not extended (original motor bogie configuration) no bogie components protruded out of the side of the cavity but, with the shaft extensions, some of the components or parts of them were no longer shielded by the bogie cavity. This will lead to an 
increase in the incident flow speed impinging on those components. The sketches in Figure 16 show front views in each case. This can be used to assess the surface area of each component exposed to the side flow.
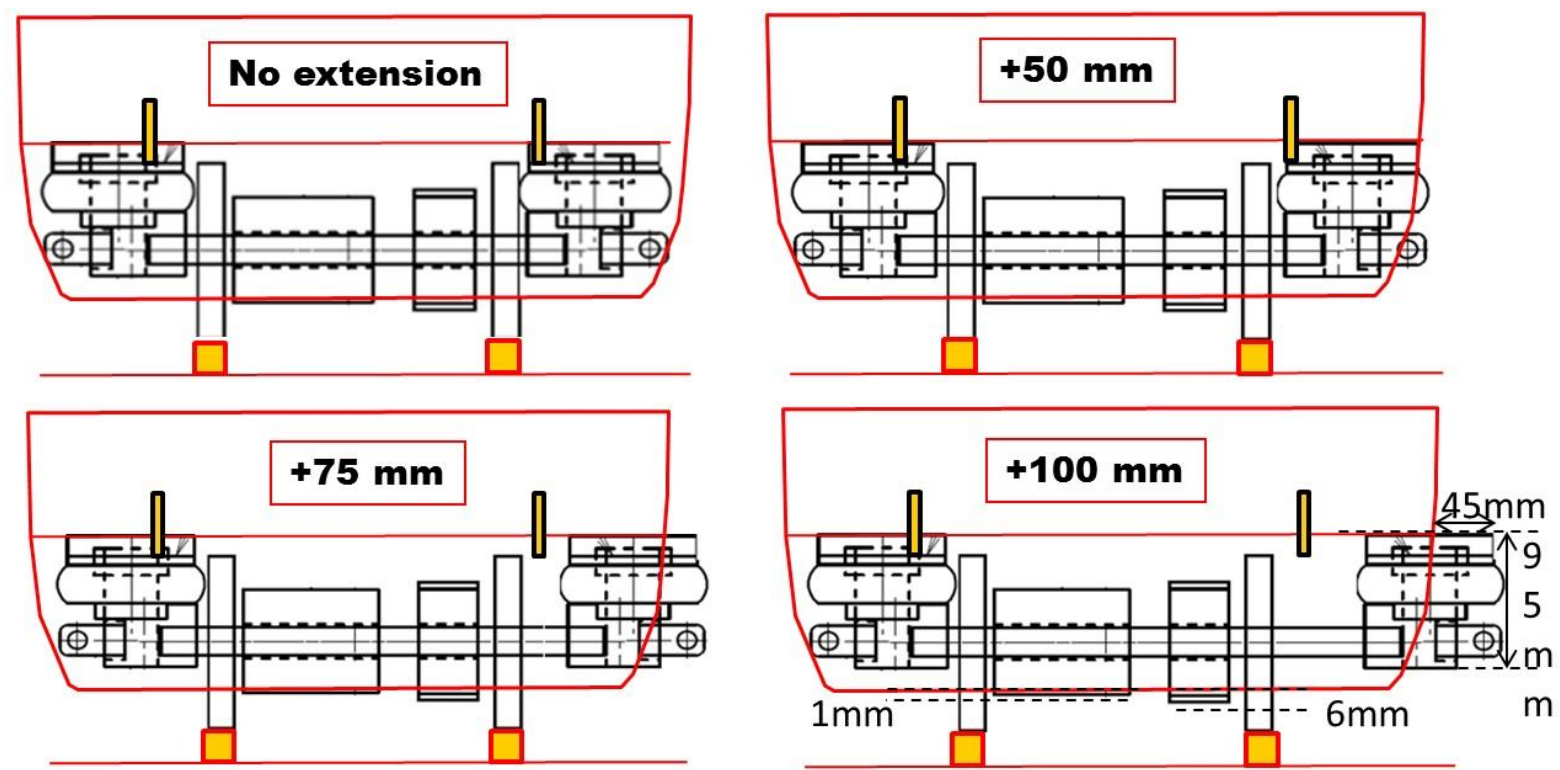

Figure 16. Sketch showing the relative position of the bogie components respect to the car body for the different shaft extensions: 50, 75 and $100 \mathrm{~mm}$ (frontal view).

The noise spectrum increases for each of the shaft extensions, as shown in Figure 17. The noise increases in all the frequency bands but the increase is smaller at low frequencies.

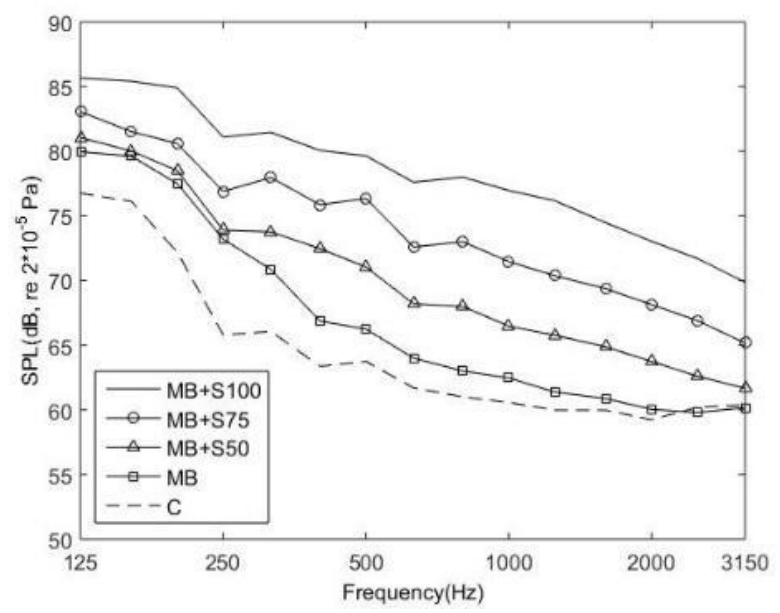

Figure 17. Noise spectra radiated by the different bogie side noise configurations and by the empty rounded bogie cavity C (shown in Figure 6(c)). Flow speed of $89 \mathrm{~m} / \mathrm{s}$ and full scale frequency range. 
Figure 18 shows the noise maps for the $1 \mathrm{kHz} 1 / 3$ octave band for the four configurations. For the configuration MB with no shaft extension the noise source is located around the bogie region but it is not possible to pinpoint a specific component as the most significant noise source. When the wheel shafts are extended by $50 \mathrm{~mm}$ (Figure 18(b)) the lateral damper is found to be the main noise source. This is exposed to the side flow, see Figure 16, leading to a moderate increase of the noise. With a shaft extension of $75 \mathrm{~mm}$, equal contributions are found for the lateral damper and the front of the side frame, while the latter is the dominant noise source for a shaft extension of $100 \mathrm{~mm}$. In these cases more of the bogie components protrude outside the bogie cavity, see Figure 16, and consequently the noise increases. These results show the importance of the relative position of the bogie components with respect to the bogie cavity.

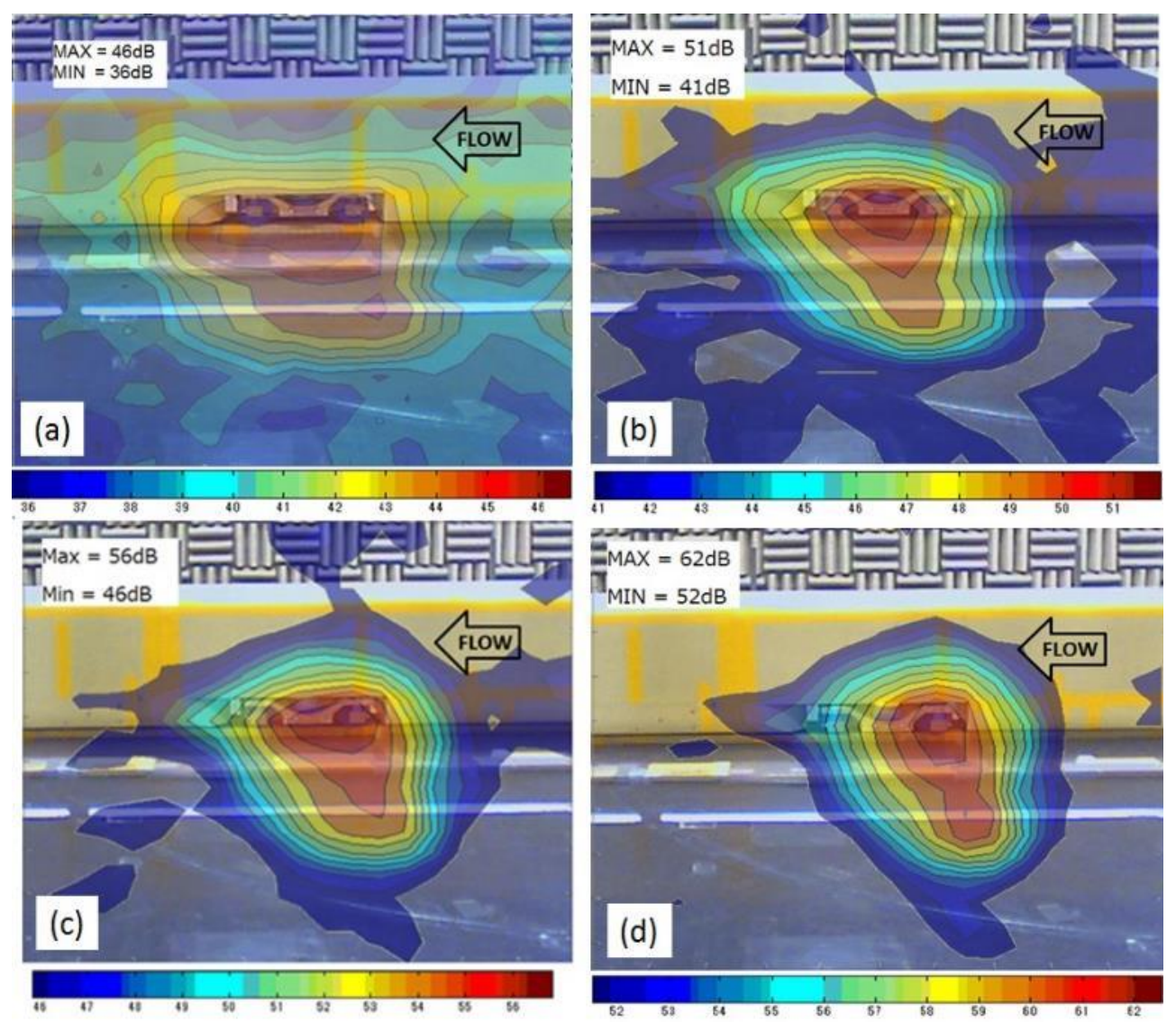

Figure 18. Noise maps for the full scale $1 / 3$ octave band of $1 \mathrm{kHz}$ for different lengths of the bogie axle extensions: $50(\mathrm{MB}+\mathrm{S} 50), 75(\mathrm{MB}+\mathrm{S} 75)$ and $100 \mathrm{~mm}(\mathrm{MB}+\mathrm{S} 100)$. Flow speed of $89 \mathrm{~m} / \mathrm{s}$. (a) Motor bogie standard configuration (MB). (b) MB+S50. (c) MB+S75. (d) MB+S100. 


\subsection{Speed exponent}

The speed exponent $\alpha$ was calculated as the slope of the OASPL (from 160 to $3150 \mathrm{~Hz}$, full scale) against the flow speed obtained from a least square fit. The graphs of OASPL against speed are shown in Figure 19 for the motor bogie configuration MB, and the three configurations with shaft extensions, $\mathrm{MB}+\mathrm{S} 50, \mathrm{MB}+\mathrm{S} 75$ and $\mathrm{MB}+\mathrm{S} 100$. In this case the background noise correction was applied to the data. It can be inferred that the speed exponent is not strongly dependent on the relative position of the bogie inside the cavity. The speed exponents obtained are close to 6, as expected for a theoretical dipole source [15].

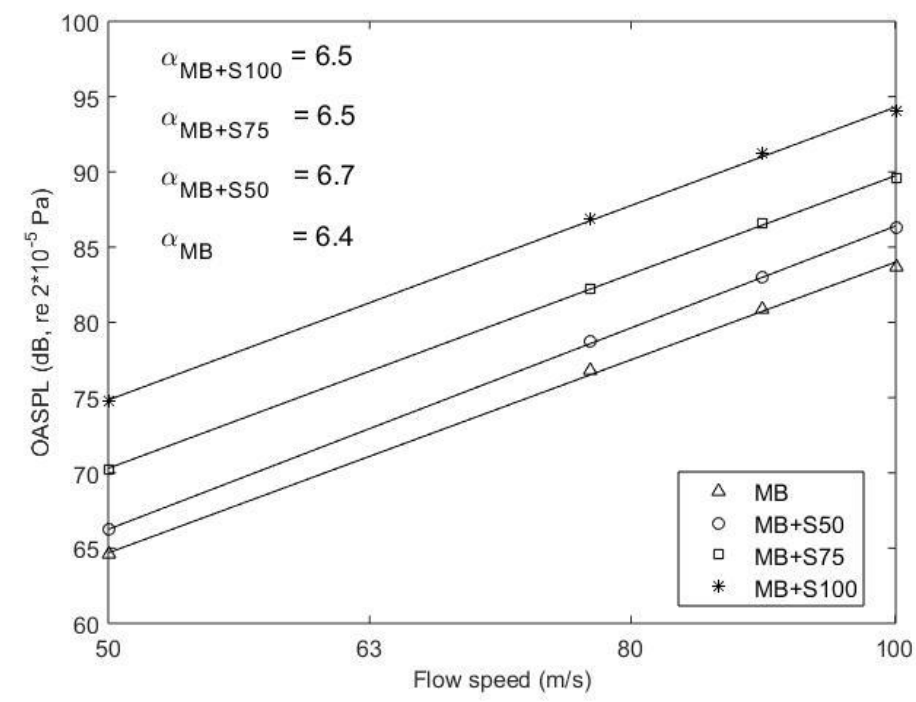

Figure 19. Dependence of the OASPL on the flow speed for the standard motor bogie (MB) and the configurations with the shafts extended by $50(\mathrm{MB}+50), 75(\mathrm{MB}+75)$ and $100 \mathrm{~mm}(\mathrm{MB}+100)$.

Figure 20 shows the noise spectra for each speed after applying a correction of $54 \log _{10}(U)$ and plotting against a Strouhal number for $D=1$. Good agreement is seen between the collapsed noise spectra for the motor bogie configuration $\mathrm{MB}$ for $\alpha=5.4$. The slope of the collapsed one-third octave spectra corresponds approximately to $10 \mathrm{~dB} /$ decade, which accounts for the difference between the value of $\alpha$ found here and in Figure 19. Similarly for the configuration with $100 \mathrm{~mm}$ shaft extension $\mathrm{MB}+\mathrm{S} 100$ good agreement is seen for $\alpha=5.5$. 

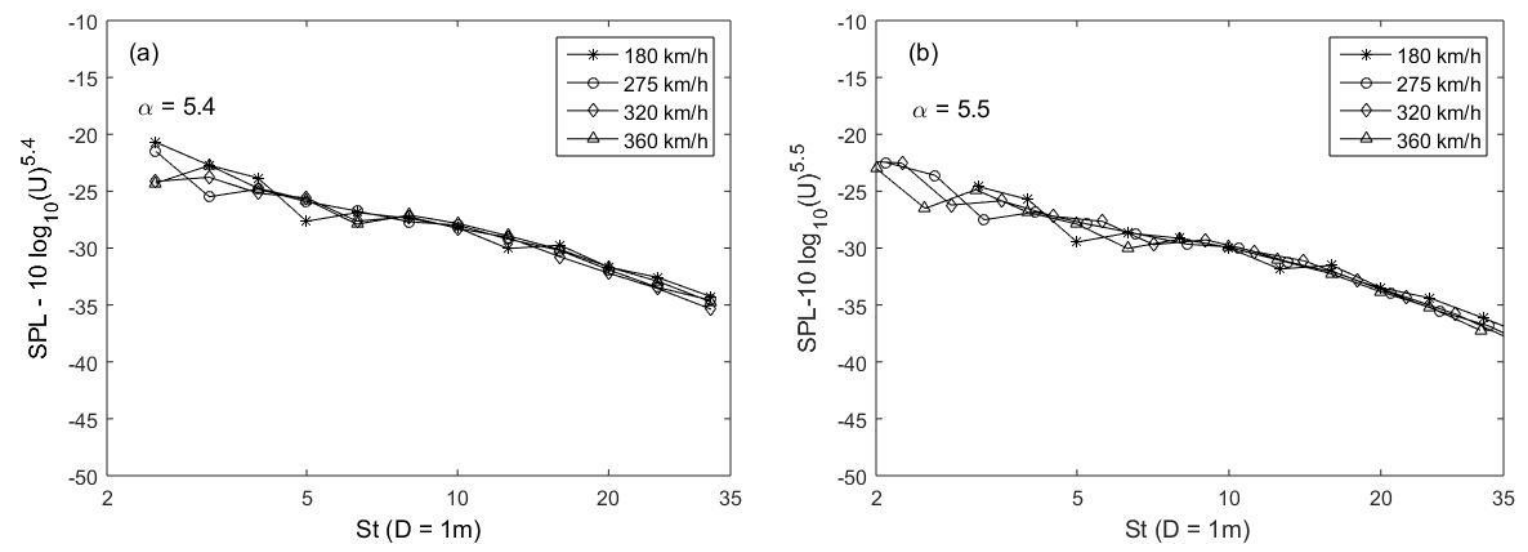

Figure 20. Noise spectra collapsed in the Strouhal number range. (a) Motor bogie configuration MB. (b) Motor bogie with a shaft extension of $100 \mathrm{~mm} \mathrm{MB+S100.}$

\subsection{Directivity}

Directivity measurements were obtained by using seven omnidirectional microphones. These were mounted parallel to the flow direction at a height of $50 \mathrm{~cm}$ above the ground plate. Angles of radiation between $-45^{\circ}$ and $45^{\circ}$ in steps of $15^{\circ}$ were covered, as shown in Figure 21 . For the directivity measurements the bogie configuration with the axles extended by $100 \mathrm{~mm}$, as shown in Figure 16, was used in order to maximise the signal-to-noise ratio. It is assumed that the directivity of the noise radiated by the side components is representative of the noise radiated by the bogie itself.

Figure 22 shows the OASPL at different angles with respect to the bogie centre for different flow speeds. For this measurement configuration, the signal-to-noise ratio was not sufficient for frequencies below $800 \mathrm{~Hz}$, reducing the effective frequency range to values between 800 and 3,150 Hz (full scale). The results have also been corrected by subtracting the background noise corresponding to the empty rounded cavity (configuration C). The results have also been adjusted to remove the effect of the differences in the distance between the bogie centre and each of the microphones assuming free-field conditions and spherical spreading. 

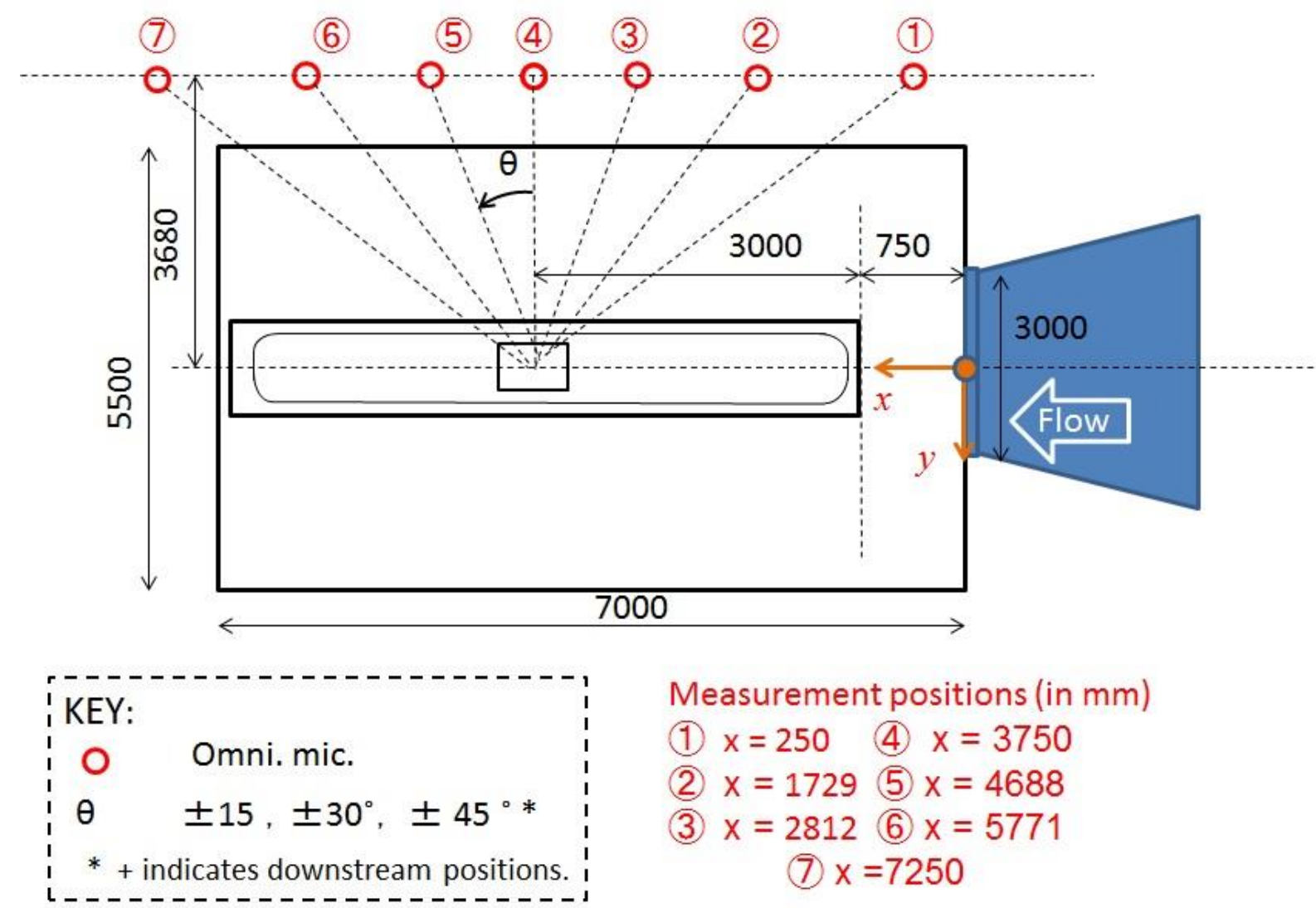

Measurement positions (in $\mathrm{mm}$ )

(1) $x=250$ (4) $x=3750$

(2) $x=1729$ (5) $x=4688$

(3) $x=2812$ (6) $x=5771$

(7) $x=7250$

Figure 21. Sketch of the experimental set-up for the directivity measurements.

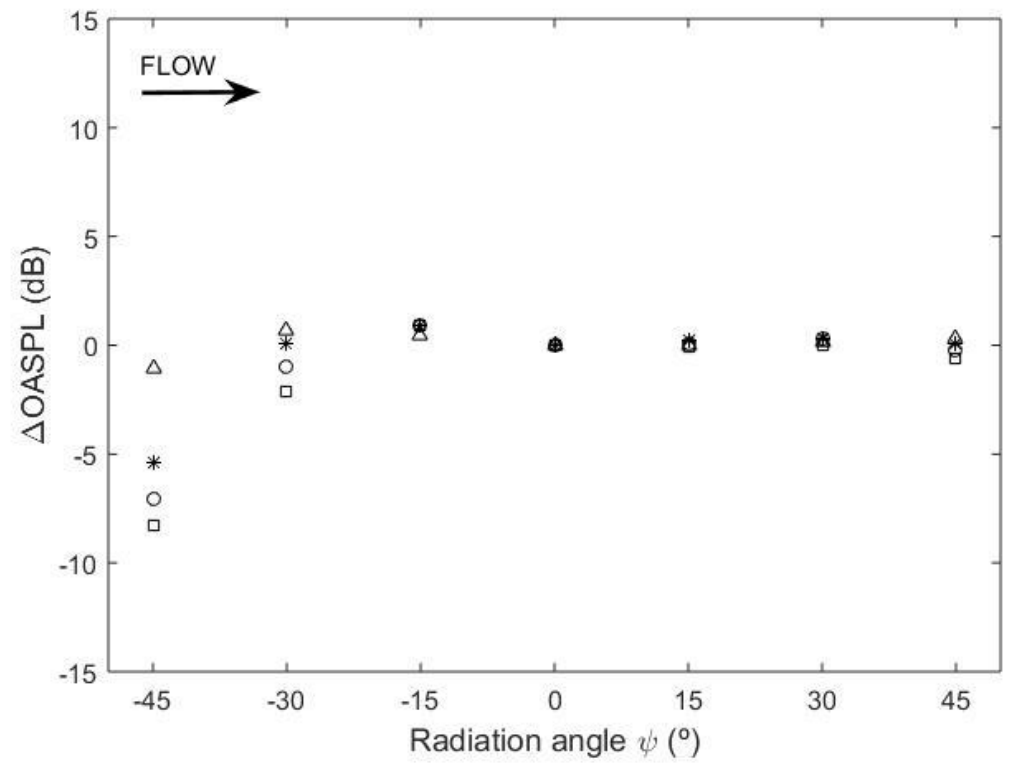

Figure 22. OASPL difference between all the microphones and microphone 4 measured for the configuration $\mathrm{MB}+\mathrm{S} 100$ for different radiation angles and different flow speeds: $\Delta 50 \mathrm{~m} / \mathrm{s}$, $* 76 \mathrm{~m} / \mathrm{s}$, ○ $89 \mathrm{~m} / \mathrm{s}$ and $\square 100 \mathrm{~m} / \mathrm{s}$. 
The changes in the noise amplitude due to the convective amplification [19], sound convection inside the jet and shear layer refraction have been neglected as the correction to be applied to account for this was less than $3 \mathrm{~dB}$.

The differences in the measured OASPL are small between microphones indicating that the directivity is nearly omnidirectional and independent of the flow speed. The OASPL measured by microphone 1 at $-45^{\circ}$ (and microphone 2 at $-30^{\circ}$ for the highest flow speeds) is significantly lower than that measured by the other microphones and this effect increases with the flow speed. Taking the bogie as the main noise source, microphone 1 is placed beyond the theoretical limit angle (around $-40^{\circ}$ for a flow speed of $89 \mathrm{~m} / \mathrm{s}$ using Amiet's method [12]) for which the sound is expected to be refracted by the shear layer, preventing it from propagating out of the jet (i.e. it is in the acoustic shadow area). Therefore, the noise from the bogie area is not detected by microphone 1 . The directivity was also found to be largely independent of frequency [18].

\section{Simple assessment method for the effect of the bogie lateral position}

The noise generated by the interaction of an incoming air flow and a solid body is equivalent to a distribution of dipole sources on the body's surface [15]. After applying a dimensional analysis it was shown by Curle [15] that the sound intensity I radiated by the dipole sources in the acoustic far-field is given by

$$
I \propto \rho_{0} U_{0}^{6} S d^{-2} c_{0}^{-3}
$$

where $\rho_{0}$ is the air density, $d$ is the distance between the noise source and the receiver, $\mathrm{S}$ is the solid surface area and $c_{0}$ is the speed of sound. In the present experiments the speed exponent $\alpha$ has been found to be slightly higher than the theoretical value of 6 . 
Therefore, the variation of the SPL radiated by a bogie component due to changes in the mean inflow speed from $U_{0}$ to $U$ or in the surface area exposed to the incoming flow from $S_{0}$ to $S$ can be estimated by

$$
10 \log _{10}\left(\frac{S}{S_{0}}\left(\frac{U}{U_{0}}\right)^{\alpha}\right)
$$

As an example, this approximate method can be applied to assess the variation of the noise expected due to the extension of the bogie shafts. Table 1 shows the surface area of the objects located totally or partially outside the bogie cavity and the averaged mean flow speed from Figure 14 for the configurations MB, MB+S50, MB+S75 and MB+S100. The surface area for the configuration MB is that of the parts of the wheels, motor and gear box located outside the bogie cavity, as shown in Figure 16, here called internal components. For the side flow configurations the surface area of the protruding side components for each of the configurations is added to that from the internal components. The incident flow speed for the configuration MB is averaged using the incident flow speeds $\bar{U}_{l}$ of the different components obtained from the results shown in Figure 14(a). For the side flow configurations the incident flow speed have been approximated assuming a similar increase of the flow speeds with the lateral position as that shown in Figure 14(a). The speed exponent $\bar{\alpha}$ shown in Figure 20 is used for each configuration.

Table 2 shows the results of substituting the data shown in Table 1 into equation (2). These results are compared with those extracted from Figure 18. These are averaged for frequencies between 400 and $2000 \mathrm{~Hz}$ because the results in this frequency range were found to be nearly independent of frequency. The measured and estimated increases of noise are in good agreement. Despite the assumptions applied this method provides a simple way to estimate the variation of the bogie noise allowing for the size of the bogie components and their positions with respect to the bogie cavity. 
Table 1. Average surface area $S$ and incident flow speed for the components exposed to the incoming flow (placed outside the cavity) for the bogie configurations $\mathrm{MB}, \mathrm{MB}+\mathrm{S} 50, \mathrm{MB}+\mathrm{S} 75$ and $\mathrm{MB}+\mathrm{S} 100$.

\begin{tabular}{ccccc}
\hline \multirow{2}{*}{ Configuration } & \multicolumn{2}{c}{$S$ components, $\mathrm{m}^{2}$} & $\bar{U}_{\iota} / U_{\infty}$ & $\bar{\alpha}$ \\
& Internal & Side & & \\
\hline MB & 0.067 & 0.067 & 0.67 & 6.4 \\
MB+50 & 0.067 & 0.006 & 0.69 & 6.7 \\
MB+75 & 0.067 & 0.047 & 0.78 & 6.5 \\
MB+100 & 0.067 & 0.128 & 0.90 & 6.5 \\
\hline
\end{tabular}

Table 2. SPL average difference when the configurations $\mathrm{MB}+\mathrm{S} 50, \mathrm{MB}+\mathrm{S} 75$ and $\mathrm{MB}+\mathrm{S} 100$ are compared with the configuration MB for the different flow speeds. The estimated SPL difference is also shown for a flow speed of $89 \mathrm{~m} / \mathrm{s}$.

\begin{tabular}{|c|c|c|c|c|}
\hline \multirow{2}{*}{\multicolumn{2}{|c|}{$\begin{array}{l}\text { Flow speed } \\
\qquad(\mathrm{m} / \mathrm{s})\end{array}$}} & \multicolumn{3}{|c|}{$\Delta \mathrm{SPL}(\mathrm{dB})$, side configurations - motor bogie $(\mathrm{MB})$} \\
\hline & & $\mathrm{MB}+\mathrm{S} 50$ & $\mathrm{MB}+\mathrm{S} 75$ & $\mathrm{MB}+\mathrm{S} 100$ \\
\hline \multirow{5}{*}{ Measured } & 50 & 5.2 & 9.7 & 14.5 \\
\hline & 76 & 4.4 & 9.4 & 14.0 \\
\hline & 89 & 4.5 & 9.3 & 13.8 \\
\hline & 100 & 4.3 & 9.2 & 13.6 \\
\hline & Average & 4.3 & 9.2 & 13.6 \\
\hline Estimated, Eq. (2) & 89 & 6.2 & 8.3 & 14.7 \\
\hline
\end{tabular}

However, this method is limited in its ability to assess the effect of changing the geometry of the bogie components as this may modify the radiation efficiency, spectral shape or the speed exponent of the noise from the component.

\section{Conclusions}

The aerodynamic noise from a train bogie has been investigated using noise measurements carried out in the large-scale low-noise anechoic wind tunnel at Maibara, Japan with a 1/7 scale train car and bogie model. Tests were carried out for speeds between 50 and $100 \mathrm{~m} / \mathrm{s}$. It has been shown that the local inflow speed has a significant effect on the noise radiation. Consequently, only the bogie components or parts of them that are exposed to the incoming flow and not shielded by the bogie cavity are significant for the noise radiation. For the train 
model used no significant differences were found between the trailer and motor bogie configurations. The trailing edge of the bogie cavity is a significant source of noise. In the present experiments, its influence has been reduced by rounding the trailing edge to allow the bogie itself to be studied in more detail.

The overall SPL follows a speed exponent between 6.4 and 6.7 for the different bogie configurations. The spectrum is fairly broad-band, the one-third octave spectrum falling with frequency at a rate of approximately $10 \mathrm{~dB} /$ decade over the range $125-3150 \mathrm{~Hz}$ (full scale). The directivity is approximately omnidirectional to within $+/-1 \mathrm{~dB}$.

The position of the bogie along the train affects the noise from the bogie area as the incoming flow speed is higher for the leading car of the train than for a car mid-way along the train. Differences of around 2.5-3.5 dB were found between the noise measurements carried out with the flow conditions representing the leading and the middle car configurations. As the leading car configuration represents the second bogie of the train, it can be expected that the first bogie will have an even higher noise level.

The noise levels increased considerably when the bogie side-frame was positioned further out from the centreline so that it was exposed to the incoming flow. This noise increase can be estimated approximately using the surface areas exposed to the flow and the mean flow speeds at different distances from the train wall.

\section{Acknowledgements}

The work presented in this paper was carried out during a six month placement at the RTRI, Tokyo, Japan, under the Pre/Postdoctoral Fellowship for Foreign Researchers Award given by the Japan Society for the Promotion of Science (JSPS). The authors are grateful to the JSPS for the funding provided and to the RTRI for making the Maibara wind tunnel available. 


\section{References}

1 Talotte C. Aerodynamic noise: a critical survey. J Sound Vib. 2000;231(3):549-562. DOI:10.1006/jsvi.1999.2544.

2 Thompson D. Railway Noise and Vibration: Mechanisms, Modelling and Means of Control. Elsevier: Oxford; 2008.

3 Mellet C, Létourneaux F, Poisson F. et al. High speed train noise emission: Latest investigation of the aerodynamic/rolling noise contribution. J Sound Vib. 2006;293(3):535-546. DOI:10.1016/j.jsv.2005.08.069.

4 Poisson F, Gautier PE, Letourneaux F. Noise Sources for High Speed Trains: A Review of Results in the TGV Case. Notes Num Fluid Mech Multidiscip Des 2008;99:71-77.

5 Fremion N, Vincent N, Jacob M, et al. Aerodynamic noise radiated by the intercoach spacing and the bogie of a high-speed train. J Sound Vib. 2000;231(3):577-593. DOI:10.1006/jsvi.1999.2546.

6 Lauterbach A, Ehrenfried K, Loose S, et al. Microphone array wind tunnel measurements of Reynolds number effects in high-speed train aeroacoustics. Int $\mathbf{J}$ Aeroacoust. 2012;11(4):35-62. DOI:10.1260/1475-472X.11.3-4.411.

7 Yamazaki N, Ido A. Evaluation methods for aerodynamic noise from a high-speed train bogie in a wind tunnel test. In: Inter noise 2011, Osaka, Japan.

8 Yamazaki N, Kitagawa T, Uda T, et al. Evaluation Method for Aerodynamic Noise Generated from the Lower Part of Cars in Consideration of the Characteristics of Under-floor Flows on Shinkansen Trains. QR of RTRI. 2016; 57(1):61-68.

9 Thompson DJ, Latorre Iglesias E, Liu X, et al. Recent developments in the prediction and control of aerodynamic noise from high-speed trains. Int J Rail Transportat. 2015;3(3):119-150. DOI:10.1080/23248378.2015.1052996.

10 Maeda T, Kondo Y. RTRI's large-scale low-noise wind tunnel and wind tunnel tests. QR of RTRI. 2001; 42(2):65-70.

11 Amiet RK. Refraction of sound by a shear layer. J Sound Vib. 1978;58(4):467-482. DOI:10.1016/0022-460X(78)90353-X.

12 Sulaiman Z. Effect of open-jet shear layers on aeroacoustic wind tunnel measurements [master's thesis]. Delft: Delft University of Technology; 2009. 
13 Lauterbach A, Ehrenfried K, Kröber S, et al. Microphone array measurements on high-speed trains in wind tunnels. In: Berlin Beamforming Conference, Berlin, Germany; 2010.

14 Sijtsma P, Oerlemans S, Tibbe T, Berkefeld T, and Spehr C. Spectral broadening by shear layers of open jet wind tunnels. Technical Report NLR-TP-2014-258, National Aerospace Laboratory NLR, 2014.

15 Curle N. The influence of solid boundaries upon aerodynamic sound. P Roy Soc Lond A Mat. 1955;231(1187):505-514. DOI:10.1098/rspa.1955.0191.

16 Latorre Iglesias E. Component-based model to predict aerodynamic noise from highspeed trains [dissertation]. Southampton: University of Southampton; 2015.

17 Christensen JJ, Hald J. Beamforming no.1. Denmark: Brüel \& Kjaer; 2004. (Technical report BV0056-11).

18 Iwasaki M, Ido A, Yamazaki N, et al. Measurement of under-floor flow of Shinkansen train passing on slab track. In: J-rail, Japan; 2013. Japanese.

19 Dowling AP, Ffowcs Williams JE. Sound and Sources of Sound. Chichester: Horwood; 1983. 